Eberhard Schwark

\title{
Wirtschaftsordnung und Sozialstaatsprinzip
}

Antrittsvorlesung

25. Januar 1996

Humboldt-Universität zu Berlin

Juristische Fakultät 
Herausgeberin:

Die Präsidentin der Humboldt-Universität zu Berlin Prof. Dr. Marlis Dürkop

Copyright: Alle Rechte liegen beim Verfasser

Redaktion:

Gudrun Kramer

Forschungsabteilung der Humboldt-Universität

Unter den Linden 6

10099 Berlin

Herstellung:

Linie DREI, Agentur für Satz und Grafik

Wühlischstr. 33

10245 Berlin

Heft 69

Redaktionsschluß: 05. 06. 1996 
$\mathrm{Vo}$ or nahezu 70 Jahren, am 23. Juni 1926, hat John Maynard Keynes an der Berliner Universität, der heutigen Humboldt-Universität, einen denkwürdigen Vortrag gehalten, der unter dem Titel „Das Ende des laissez-faire“ im Duncker \& Humblot-Verlag erschienen ist. Keynes machte seinerzeit darauf aufmerksam, daß sich bei den klassischen Denkern des Individualismus und des Freihandels - genannt seien nur Hume und Adam Smith - nichts von der trivialen Variante der laissez-faire-Formel finde ${ }^{1}$, sondern erst die Utilitaristen (Payne, Bentham) den Egoismus des einzelnen mit dem größten Glück der größtmöglichen Zahl zu vereinen wußten. Der Staatsphilosoph, so Keynes damals sarkastisch, konnte nunmehr dem Geschäftsmann das Feld räumen - denn dieser vermochte das Summum Bonum des Philosophen zu erreichen, wenn er lediglich seinen eigenen Vorteil verfolgte. ${ }^{2}$

Dieser „Pudding“ (ein Ausdruck von Keynes), dessen Bekömmlichkeit schon von der Zeit John Stuart Mills an angezweifelt wur$\mathrm{de}^{3}$, habe sich inzwischen als ungenießbar herausgestellt; aus den Prinzipien der Nationalökonomie lasse er sich jedenfalls nicht ableiten. Vielmehr müßten die Agenda und Non agenda des Staates neu bestimmt werden. ${ }^{4}$ Heute mehren sich erneut die Anzeichen für eine Gegenbewegung in Richtung Abbau staatlicher Aktivitäten, Reprivatisierung von Sektoren, die in einer langen Entwicklung zu öffentlichen Aufgaben geworden waren, Rückzug des Staates aus fiskalischer oder bisher der Daseinsvorsorge zugeordneter Unternehmenstätigkeit, teilweiser Ersetzung des öffentlichen sozialen Netzes der Bevölkerung durch private Beteiligung an Krankheitskosten und Altersvorsorge etc. Haben wir es ideengeschichtlich und politökonomisch also mit einem Wiedererstarken des laissez-faire-Gedankens zu tun, nachdem die gegnerischen Ideen, der Protektionismus und der marxistische Sozialismus, jedenfalls in den westlichen Industriestaaten, abgedankt haben? 
Wenn das so ist, wenn also, sei es wegen abnehmender finanzieller Leistungsfähigkeit des Staates und der Steuerzahler ${ }^{5}$, sei es, weil Friedrich A. v. Hayeks Kampf gegen konservative wie proletarische Sozialismen in der Tat den Weg in die Knechtschaft zu verhindern scheint, stellt sich die Frage des grundgesetzlichen Sozialstaatsprinzips und seiner Bedeutung für die Wirtschaftsordnung erneut.

Das damit angezeigte Thema ist zwar ein juristisches, kann aber ohne die ökonomische Entwicklungsgeschichte, den staatsphilosophischen Hintergrund, die soziale Frage des 19. Jahrhunderts: die Arbeiterfrage, um nur die wichtigsten Bezugspunkte zu nennen, nicht verstanden werden. Dieser historische Rahmen muß hier als bekannt vorausgesetzt werden.

\section{Eingrenzung des Themas, Begriffliches}

Die Bundesrepublik ist ein demokratischer und sozialer Bundesstaat, heißt es in Art. 20 Abs. 1 GG; in Art. 28 Abs. 1 GG lesen wir: Die verfassungsmäßige Ordnung in den Ländern muß den Grundsätzen des republikanischen, demokratischen und sozialen Rechtsstaats i. S. dieses Grundgesetzes entsprechen. Allein aus dem dem Wort Bundesstaat bzw. Rechtsstaat beigefügten Adjektiv wird das verfassungsrechtliche Sozialstaatsprinzip abgeleitet; wir haben es mithin mit einer ganz unbestimmten Generalklausel, einer nicht detaillierten Blankettnorm zu tun. ${ }^{6}$ Es verwundert deshalb nicht, daß Roman Herzog dieses Prinzip als das umstrittenste verfassungsrechtliche Prinzip der gesamten politischen Landschaft der Bundesrepublik bezeichnet, dessen inhaltliche Konkretisierung der Verfassungsgerichtsbarkeit die größten Schwierigkeiten bereite. ${ }^{7}$ Hinzu kommt, daß auch die Fachwelt auf Interpretationsversuche nicht selten so reagiert, wie der Zeitungsleser eines Leitartikels. Hat dieser ein Reizwort gelesen, genügt ihm das. Er glaubt dann zu wissen, wes Geistes Kind hinter dem ganzen Artikel steckt.

Die Vertreter des Wirtschaftsrechts und des Öffentlichen Rechts haben gleichwohl immer wieder versucht, den allgemeinen 
Rechtsbegriff „Sozialstaat“ auszulegen und zu konkretisieren. Blieb nach Herbert Krüger ${ }^{8}$ noch im Dunkeln, was der Sozialstaat ist und meint, so wagte bereits Ballerstedt den Satz, daß eine freiheitlich-soziale Wirtschaftsverfassung die Erfüllung des Sozialstaatsgebots in der Wirtschaftsordnung sei. ${ }^{9}$ Gewöhnlich werden Elemente der Sozialstaatlichkeit im Wege einer historisch-politischen Darstellung dessen, was die soziale Bewegung zur Mehrung des gesellschaftlichen Wohlstandes erreicht hat ${ }^{10}$ oder durch eine enzyklopädische Beschreibung der Regelungsbereiche, in denen sich die Sozialstaatsklausel entfaltet ${ }^{11}$, gewonnen, um so über eine Wertentscheidung hinaus zu einem Rechtssatzcharakter ${ }^{12}$ des Prinzips zu gelangen.

Das Bundesverfassungsgericht, das nicht dazu berufen ist, Handbücher zu schreiben, hat sich sehr zurückhaltend geäußert: Ein Kernsatz in der Begründung eines im 22. Band ${ }^{13}$ abgedruckten Urteils lautet: „Art. 20 Abs. 1 GG bestimmt nur das „Was“, das Ziel, die gerechte Sozialordnung; er läßt aber das ,Wie“, d. h. für die Erreichung des Ziels alle Wege offen“. Wird aber, wie mit diesem Satz, nur die „Zweckkonditionierung " ${ }^{\text {"14 }}$ des Rechtsprinzips vorgegeben, werden die mit einem allgemeinen Rechtsbegriff regelmäßig verbundenen Defizite der Instabilität verstärkt. Nimmt man hinzu, daß das Prinzip Sozialstaat ,nach oben offen ist ${ }^{\text {" } 15}$, also ein Aufruf zur Aktion ist und in Richtung auf mehr soziale Sicherheit und Hebung der „sozialen Normalität“ (Zacher) zielt, was im Zweifel zu mehr Staatsaufgaben führt ${ }^{16}$, erkennt man das gefährliche Potential, das ein für die gesellschaftlichen, sozialen und finanziellen Realitäten blindes Sozialstaatsprinzip für eine freiheitliche Wirtschaftsordnung haben kann. Erst wenn der umgestaltende Aktionismus, den das „Was“ des Sozialstaats vorgibt, durch das „Wie“ der wirtschaftsverfassungsrechtlichen Vorgaben eingefangen wird, nimmt das Sozialstaatsprinzip in beiden Richtungen justitiable Gestalt an: Es kommt nicht zu einer einseitigen Verteilung der Beweislast zugunsten einer sozialen Angleichung. ${ }^{17}$ Auf die Wirtschaftsordnung bezogen, hat dies schon Ballerstedt als die ständige Aufgabe beschrieben, das unbequeme Dreieck der drei Grundanliegen Wirtschaftsfreiheit - Vollbeschäftigung - Stabilität des Geldwertes auszubalancieren..$^{18}$ Der 
Vorwurf, der hier dem Bundesverfassungsgericht gemacht wird, wiegt weniger schwer, wenn man sich vor Augen führt, daß das Gericht in einer Reihe von Einzelentscheidungen jedenfalls mittelbar zur Konkretisierung des „Wie“ beigetragen hat. Wenn das Gericht zwar keinen Zahlbetrag für soziale Leistungen nennt, aber es für zwingend erklärt, daß der Staat die Mindestvoraussetzungen für ein menschenwürdiges Dasein seiner Bürger schafft ${ }^{19}$, läßt sich aufgrund eines Warenkorbes unschwer errechnen, was dies für die Versorgung mit Lebensmitteln bedeutet, ohne daß sich daraus freilich eine positive Justitiabilität des Sozialstaatsprinzips ergibt. ${ }^{20}$ Wenn dieses Prinzip als Auslegungsrichtlinie für das Grundgesetz und andere Gesetze zu dienen hat, lassen sich daran steuerliche Regelungen zum Konjunkturzuschlag ${ }^{21}$ und zur Objektsteuer messen ${ }^{22}$ und ist bei staatlichen Leistungen eine Differenzierung nach dem Grad der sozialen Schutzbedürftigkeit des Empfängers erlaubt. ${ }^{23}$ So hat das Bundesverfassungsgericht das Gesetz zur Überführung der Anteilsrechte der Volkswagen $\mathrm{GmbH}$ in private Hand vom 27.7.1960, das besondere Regelungen zugunsten einkommensschwacher Schichten enthielt, für verfassungsgemäß gehalten. ${ }^{24} \mathrm{Zum}$ Preisgesetz hat das Gesetz judiziert, eine gesetzliche Regelung, die es ermögliche, aus gesamtwirtschaftlichen und sozialen Gründen die zum Nutzen des allgemeinen Wohls erforderlichen Maßnahmen zu treffen, entspreche dem Sozialstaatsprinzip. ${ }^{25}$ Da die Kleinaktionäre keine sozial abgrenzbare Gruppe bildeten, widerspreche andererseits die Umwandlung einer AG durch Mehrheitsbeschluß gem. § 12 Umwandlungsgesetz a. F., die zur Expropriierung der Kleinaktionäre führt, nicht dem Sozialstaatsprinzip. ${ }^{26}$ Schließlich ist auf die wichtige Entscheidung des Gerichts aus dem Jahre 1992 hinzuweisen, mit der es die Steuerfreiheit des erarbeiteten Existenzminimums dekretiert hat. ${ }^{27}$ In dem im Schwerpunkt auf andere Verfassungsbestimmungen (Art. 2, 6, 12, 14 GG) gestützten Urteil stellt das Gericht fest, daß das steuerfreie Existenzminimum jedenfalls nicht unter den aufgrund sozialstaatlicher Fürsorge zu erbringenden Leistungen liegen dürfe.

Die bisherigen Bemühungen zur Konkretisierung des Sozialstaatsprinzips, wie immer man ihren Erfolg auch beurteilen mag, 
lassen für unser Thema eine wichtige Eingrenzung zu, die ein sonst kaum zu vermeidendes Ausufern verhindert. Wer sich auf das Verhältnis von Sozialstaatsprinzip und Wirtschaftsordnung konzentriert, kann jenen historisch im Vordergrund stehenden Strang des Sozialstaats außer acht lassen, der sich in der Abwehr von Not und Armut des einzelnen, d. h. in allen vorsorgenden, entschädigenden, helfenden oder fördernden Leistungen der Sozialsysteme ausdrückt. Denn hier, beim Arbeitslosengeld, dem Wohngeld, der Sozialfürsorge oder der Resozialisierung geht es um Vorsorge für das Individuum und seine Familie oder, wie Zacher das nennt, um den mikrosozialen Ansatz. ${ }^{28}$ Wer nach Auswirkungen des Sozialstaatsprinzips auf die Wirtschaftsordnung fragt, meint dagegen den auf die Gesamtwirtschaft bezogenen makrosozialen Ansatz; dieser gehört zum Wirtschaftsrecht, jener ist sozialrechtlich geprägt. Beide Wege müssen nebeneinander stehen. Denn wer die makroökonomische Seite ebenso stark in den Prozeß der Verteilung einbindet wie die mikrosoziale, hebt die Autonomie der Wirtschaftssubjekte auf; die verfassungsrechtliche Unzulässigkeit einer solchen umfassenden Planung wird später zu begründen sein.

Unter Wirtschaftsordnung wird hier die Gesamtheit aller grundlegenden (normativen) Regeln für Aufbau und Ablauf des wirtschaftlichen Geschehens verstanden. ${ }^{29}$ Gemeint sind damit also weder die tatsächlichen Formen, in denen der Wirtschaftsprozeß in concreto abläuft, ${ }^{30}$ noch die Wirtschaftssysteme, die sich auf konstitutive Grundformen der Wirtschaft, wie reine Marktwirtschaft oder reine Planwirtschaft, beziehen. Wirtschaftsordnung im hier verstandenen Sinne und Wirtschaftsverfassung werden oft synonym gebraucht. ${ }^{31}$ Die häufig benutzte Bezeichnung Wirtschaftsverfassung erscheint jedoch mißverständlich; sie suggeriert, daß das Grundgesetz eine Wirtschaftsverfassung im materiellen Sinne vorgibt; in Wahrheit enthält es nur Einzelbestimmungen, die auch, in wenigen Fällen in erster Linie, für die Wirtschaft gelten. 


\section{Sozialstaatsprinzip und Wirtschaftsordnung unter systematischem Blickwinkel}

Auf der Linie der juristischen Kanones ${ }^{32}$ fortschreitend, ist nunmehr unter systematischem Blickwinkel nach dem Verhältnis von Wirtschaftsordnung und Sozialstaatsprinzip zu fragen. Als grundlegend erweist sich die Verknüpfung von Sozialstaat und Rechtsstaat in Art. 28 GG. Damit wird auf den Rechtsstaat des GG im materiellen Sinne, vor allem die grundrechtlichen Freiheitspositionen verwiesen. ${ }^{33}$ Die Freiheit der wirtschaftlichen Betätigung findet in Art. 2, 12 und 14 GG ihren Ausdruck ${ }^{34}$, die Freiheit, sich unternehmerisch in der Form von Handelsgesellschaften zu betätigen, in Art. $9 \mathrm{GG}^{35}$, ebenso die kollektive Wahrnehmung von Interessen durch die Tarifvertragsparteien (Art. 9 Abs. 3 GG). Freiheitlicher Rechtsstaat und Sozialstaat können nicht in einen halben Rechtsstaat und einen halben Sozialstaat zerlegt werden in der stillen Hoffnung, daß sich daraus schon ein ganzer Sozialrechtsstaat ergebe. ${ }^{36}$ Sie stehen vielmehr in einem Spannungsverhältnis, das von Fall zu Fall pragmatisch aufzulösen ist. ${ }^{37}$ In Goethes „,Maximen und Reflexionen" lesen wir den Satz: Gesetzgeber und Revolutionärs, die Gleichsein und Freiheit zugleich versprechen, sind Phantasten oder Scharlatans. ${ }^{38}$ Aus heutiger Sicht verfehlt dieser Satz das GG, verkennt er doch, daß beide Prinzipien, das auf die Beseitigung von Ungleichheit zielende Sozialstaatsprinzip ${ }^{39}$ und die Freiheit der wirtschaftlichen Betätigung beide zusammen und keines auf Kosten des anderen zu verwirklichen sind. Aus der Spannungslage von Sozialstaatsprinzip und Freiheit, keineswegs eine ,platte Antinomie" oder „verfassungsrechtliche Trivialität", wie Kittner meint ${ }^{40}$, lassen sich erste Konsequenzen für die grundgesetzlich zulässige Wirtschaftsordnung ableiten:

Wenn sich die Verfassung für ein Gegenüber von Staat und Gesellschaft entschieden hat ${ }^{41}$, bedeutet dies das Verbot einer ,staatlichen Kommandowirtschaft" (Dürig), die die Gesellschaft unter dem Deckmantel des Sozialen in den Staat integriert. Dabei ist die (völlige) Ineffizienz administrativer Planwirtschaften, die zu einem niedrigen Einkommensniveau der gesamten Bevölkerung und zu einer Zerstörung des privaten Unternehmensvermögens führt, kein Grund dafür, diese Wirtschaftsform rechtlich zu per- 
horreszieren. Der Grund für ihre Verfassungswidrigkeit liegt vielmehr im Kern darin, daß die Planwirtschaft die freie wirtschaftliche Betätigung, von kleinen Randzonen abgesehen, ausschließt und damit in einem wichtigen Teilbereich die Entfaltung der menschlichen Persönlichkeit verhindert. Daß damit zumeist auch eine Vernachlässigung von Gemeinschaftsgütern, wie der Umwelt, einhergeht, ist akzidentiell; sie zeigt nur die Perfidie solcher Systeme im konkreten Fall, ist für sie aber nicht konstitutiv. Und es ist eine Ironie der menschlichen Natur, daß Menschen, die lange Zeit in einem solchen System gelebt haben, nach der Entlassung daraus, sich nicht selten dahin zurücksehnen.

Auf der anderen Seite läßt sich auch eine Wirtschaftsordnung, die Unternehmen und Individuen frei schalten und walten läßt, mit dem Grundgesetz nicht vereinbaren. Müller-Armack ${ }^{42}$ hat schon 1947 die Soziale Marktwirtschaft, die heute bis in die Ränder des politischen Spektrums hinein als eine freilich vage ${ }^{43}$ Umschreibung des verfassungsrechtlich Gebotenen akzeptiert ist, vom laissez-faire-Liberalismus, wie folgt, abgegrenzt:

„Es war ein folgenschwerer Fehler des wirtschaftlichen Liberalismus, die marktwirtschaftliche Verteilung schon schlechthin als sozial und politisch befriedigend anzusehen und damit die Frage der zweckmäßigen technischen Austauschform mit der Frage des sozial und staatlich Erwünschten zu verquicken. Es ergab sich daraus eine Verkehrung der echten Notwendigkeit eines sozialen Einkommensausgleichs wie einer Berücksichtigung der staatlichen Notwendigkeiten. Dies entfremdete ihm alle Kräfte, die soziale und staatliche Überzeugungen vertraten und dies hinfort nur im Gegensatz und in Ausschaltung der liberalen marktwirtschaftlichen Ordnung glaubten tun zu können.“

Daß sich bekanntlich das Bundesverfassungsgericht nicht dazu verstanden hat, das Konzept der sozialen Marktwirtschaft als die Wirtschaftsordnung des GG zu erklären ${ }^{44}$, sei nur angemerkt. Das folgt freilich nicht aus systematischen Überlegungen, etwa dem Hinweis auf den Sozialisierungsartikel Art.15 GG, den H. Ridder 
schon auf der Staatsrechtslehrertagung 1952 als eine obsolete Verfassungsnorm bezeichnet hat ${ }^{45}$, sondern daraus, daß sich darunter, je nach der Gewichtung des marktwirtschaftlichen oder des sozialen Moments auch sehr Unterschiedliches verstehen läßt. ${ }^{46}$ Zudem läßt sich die privatwirtschaftliche Wirtschaftsordnung in ihrer heutigen wandelbaren Form nicht unter der Hand zum verfassungsrechtlichen Schutzgut erklären. ${ }^{47}$

Als ein herausragendes sozialstaatliches Element der Wirtschaftsordnung wird Art. 109 Abs. 2 GG $^{48}$ genannt, der die Haushaltswirtschaft von Bund und Ländern auf das ,gesamtwirtschaftliche Gleichgewicht" verpflichtet. Darunter ist die Verwirklichung des sog. magischen Vierecks (Stabilität des Preisniveaus, hoher Beschäftigungsstand, außenwirtschaftliches Gleichgewicht, stetiges und angemessenes Wirtschaftswachstum) zu verstehen (§ 1 StabilG) ${ }^{49}$ Die damit vorgegebene Pflicht zu makroökonomischer Globalsteuerung der Wirtschaft, die sich gem. $\S 1$ StabilG freilich im Rahmen der marktwirtschaftlichen Ordnung halten soll, ist für unser Thema vor allem wegen ihrer Konkretisierung des Sozialstaatsprinzips auf dem Beschäftigungssektor von Bedeutung. ${ }^{50}$ Kittner ${ }^{51}$ hat daraus wegen der engen Verbindung der bezahlten Arbeit mit der Menschenwürde den verfassungsrechtlichen Vorrang des Ziels ,hoher Beschäftigungsstand“" gefordert. Art. 1 GG laute nämlich nicht: „Die Würde des Menschen, das Wachstum und die Deutsche Mark sind unantastbar". Das Bundesverfassungsgericht hat bereits im 21. Bande ${ }^{52}$ deutlich gemacht, bei umfangreicher und langandauernder Arbeitslosigkeit in der Bevölkerung ergebe sich kein Freibrief für politische Untätigkeit unter Verweis auf die Wachstumskräfte der Wirtschaft, wenn die soziale Gerechtigkeit Aktivitäten gebiete. Dazu mehr im Besonderen Teil.

Unter systematischen Gesichtspunkten wären noch weitere wirtschaftsordnungsrelevante Verfassungsgrundsätze und Grundgesetzartikel anzuführen, die eine Beziehung zum Sozialstaatsprinzip aufweisen. Ich will es bei zwei Hinweisen belassen: Das demokratische Prinzip darf nicht dazu benutzt werden, die verfassungsrechtlichen Vorgaben zu unterlaufen, sei es um ein weiter- 
gehendes Sozialstaatsverständnis durchzusetzen ${ }^{53}$, sei es, um dem einfachen Gesetzgeber und der Exekutive mehr oder weniger plein pouvoir zu erteilen. ${ }^{54}$ Der Gleichheitssatz verstärkt entgegen der Auffassung vieler Vertreter des öffentlichen Rechts ${ }^{55}$ das Sozialstaatsprinzip in Richtung auf mehr Chancengleichheit ungeachtet aller wesensmäßigen Ungleichheiten, speziell in einer Marktwirtschaft. Die neue Rechtsentwicklung des Wirtschaftsrechts verfolgt dieses Ziel z. B. durch erweiterte Informationspflichten im Vermögensanlagebereich oder das strafbewehrte Verbot des Insiderhandels auf den Wertpapiermärkten. ${ }^{56}$

Als Zwischenergebnis der systematischen Überlegungen ist festzuhalten: Eine dem Sozialstaatsprinzip entsprechende Wirtschaftsordnung darf wegen der Verschränkung des Prinzips mit anderen Verfassungsbestimmungen nicht als ein die Wirtschaftsfreiheit beseitigendes System ausgebildet sein, wobei gleichgültig ist, ob dies durch umfassende Lenkung geschieht oder auch nur auf ganz überwiegenden Fiskalbesitz an den Unternehmen zurückzuführen ist. Aber auch eine laissez-faire-Wirtschaft steht nicht im Einklang mit diesem Prinzip. Vielmehr ist der Staat aufgrund dessen jedenfalls zur globalen Steuerung der Wirtschaft verpflichtet. Zur Finanzierung der hier nicht näher beleuchteten individuellen Sozialleistungen hat er erforderlichenfalls auch die der Umverteilung dienende Steuerpolitik progressiver Tarife einzusetzen. ${ }^{57}$ Auf das vage Modell der sozialen Marktwirtschaft läßt sich das Sozialstaatsprinzip nicht verpflichten. Dieses recht magere Ergebnis des AT läßt sich vielleicht durch die nähere Betrachtung dreier Problemfelder auffüllen, des Verhältnisses von Sozialstaatsprinzip und Markt, von Sozialstaatsprinzip und Beschäftigung und von Sozialstaatsprinzip und Privatrechtsordnung.

\section{Sozialstaatsprinzip und Markt}

Der Markt ist der Ort, an dem sich durch Zusammentreffen von Angebot und Nachfrage die Preise bilden; als Prozeß verstanden, lenkt er in einer arbeitsteiligen Wirtschaft die Erzeugung von Gütern im Interesse einer bestmöglichen Versorgung der Verbrau- 
cher. Wird die marktwirtschaftliche Orientierung, wie hier, als ein Stück verfassungsrechtlich gebotener Freiheit des Wirtschaftslebens verstanden, stellt sich sub specie des Sozialstaatsprinzips die Frage, in welchem Umfang Eingriffe in das Marktgeschehen zulässig oder geboten sind.

1. Wettbewerbsordnung als allein zulässige sozialstaatliche Marktsteuerung?

An erster Stelle zu nennen ist die Sicherung des Wettbewerbs, die in der Bundesrepublik vor allem durch das GWB und Art. $85 \mathrm{ff}$. des EG-Vertrages erfolgt. Sie wird heute durchgehend als Ausfluß des Sozialstaatsprinzips verstanden, weil sie Vorkehrungen gegen Machtmißbrauch trifft, den Schwächeren schützt und Marktzutrittsschranken abbaut. ${ }^{58}$ Franz Böhm und Nipperdey haben die Wettbewerbsordnung sogar als verfassungsrechtlich geboten bezeichnet. ${ }^{59}$ Angesichts der anderen Seite des Sozialstaatsprinzips, das, wie später zu zeigen sein wird, auch wettbewerbswidrige Maßnahmen zuläßt, kann dem nicht gefolgt werden. ${ }^{60}$ Es ist aber ein bleibendes Verdienst der sog. Freiburger Schule, erkannt zu haben, daß die Wirtschaftsfreiheit sich durch Bildung von Kartellen, den Ausbau der Konzernierung bis zum Wachsen zu Monopolunternehmen selbst aufhebt und deshalb ein Korrektiv in Form der Wettbewerbsordnung erfordert. Der Wettbewerb muß deshalb, wie Miksch gesagt hat ${ }^{61}$, eine staatliche Veranstaltung sein. Das Prekäre an dieser bis heute fortwirkenden Lehre beginnt dort, wo daraus zugleich das Postulat der Marktkonformität aller staatlichen Interventionen in die Wirtschaft abgeleitet wird, d. h. Wettbewerb, Preismechanismus und Investitionsfreiheit unangetastet zu bleiben haben. ${ }^{62}$ Noch auf der Wettbewerbstagung zum 25jährigen Bestehen des FIW, des dem BDI angegliederten Forschungsinstituts für Wirtschaftsverfassung und Wettbewerb, im Jahre 1986 ist es darüber zu einer aufschlußreichen Kontroverse zwischen Joseph Kardinal Höffner und Otto Schlecht, dem damaligen Staatssekretär im BMWi, gekommen. Höffner führte aus ${ }^{63}$, die Wettbewerbsfreiheit sei berechtigt und zweifellos von Nutzen (aequum et sane utile). Sie sei 
durch eine staatliche Rahmenordnung in ihrer Existenz und in ihrer Qualität (z. B. durch das UWG) zu sichern. Der Leistungswettbewerb sei aber nicht das regulative Prinzip der Wirtschaft schlechthin, sondern der Wirtschaft müsse im Blick auf das Gemeinwohl eine soziale Ausrichtung, ein humanes Leitbild gegeben werden. Den von der katholischen Soziallehre ${ }^{64}$ beeinflußten Äußerungen des studierten Ökonomen Höffner hielt Schlecht in der Diskussion entgegen, durch die Wettbewerbsordnung sei das Wirtschaften an den Märkten an Gerechtigkeitsanforderungen gebunden. ${ }^{65}$ Wenn der Staat seiner Aufgabe nachkomme, Wettbewerbsbeschränkungen zu bekämpfen, erfülle er in erforderlichem Umfang die sozialstaatlichen und ethischen Ansprüche.

Abgesehen davon, daß die rechtliche und wirtschaftspolitische Realität der Bundesrepublik ganz anders aussieht, nämlich durch Eingriffe auch prozeßpolitischer Art und Marktordnungen in Teilbereichen geprägt ist ${ }^{66}$, erschöpft sich das Sozialstaatsprinzip nicht in der Wettbewerbssicherung. ${ }^{67}$ Das ergibt sich schon aus dem Nebeneinander von Art. 74 Nr. 16 GG, der die Gesetzgebung zur Verhütung des Mißbrauchs wirtschaftlicher Machtstellung anspricht und Art. 74 Nr. 17, Art. 91a Abs. 1 Nr. 1 und 2 GG, die die Wirtschaftsförderung, d. h. Subventionierung, in zahlreichen Wirtschaftsgebieten und in Regionen in die konkurrierende Kompetenz des Bundes stellen. Die vom Bundesverfassungsgericht betonte Offenheit des Sozialstaatsprinzips gegenüber der konkreten Gestaltung der Wirtschaftsordnung (s. o. II) verbietet es, ökonomischen Zweckmäßigkeitserwägungen verfassungsrechtlichen Rang zu verschaffen.

\section{Subventionen, Strukturpolitik}

Daran schließt sich die Frage an, ob Subventionen ohne verfassungsrechtliche Beschränkung an die Wirtschaft gegeben werden können, in Einzelfällen aufgrund des Sozialstaatsprinzips sogar gegeben werden müssen. Subventionen beeinträchtigen den marktwirtschaftlichen Steuerungsprozeß über den Preis und sie stören den Wettbewerb zu Lasten derjenigen, die solche Hilfen 
nicht erhalten.$^{68}$ Ihr Umfang ist beträchtlich; in der Subventionsberichterstattung des Bundes wird für 1993 einschl. Steuervergünstigungen ein Betrag von 114 Mrd. DM genannt; in der Strukturberichterstattung der Wirtschaftsforschungsinstitute, die weitere Tranfers und die Förderung marktfernerer Bereiche einschl. der Bildung einbezieht, ist für das gleiche Jahr sogar von $216 \mathrm{Mrd}$. DM die Rede. Die rechtlichen Voraussetzungen für Subventionen, Abschöpfungen, Sonderabgaben und entsprechend wirkende steuerliche Maßnahmen auf einfach-gesetzlicher Ebene beschränken sich darauf, eine Legitimationsgrundlage, ggf. auch nur durch ein Haushaltsgesetz, zu verlangen. ${ }^{69}$ Eine Kontrolle der Fördermaßnahmen, vergleichbar mit Art. 92, 93 EG-Vertrag, gibt es im deutschen Recht nicht. ${ }^{70}$ Verfassungsrechtlich bestehen erst recht keine Beschränkungen; verfassungsrechtlich begründete Klagen nicht berücksichtigter und benachteiligter Konkurrenten gegen Einzelmaßnahmen führen nur in Einzelfällen zur Korrektur. Das landauf, landab erhobene Petitum, Subventionen wegen ihrer für den Wettbewerb nachteiligen Folgen zu streichen, findet mithin im geschriebenen Recht der Bundesrepublik keine Stütze.

Im Kontext des Sozialstaatsprinzips geht es ohnehin um die entgegengesetzte Frage: Was spricht zulässigerweise oder zwingend für Subventionen? An erster Stelle ist die sozialpolitische Abfederung von wirtschaftlichem Strukturwandel zu nennen. Als Beispiele seien die Förderung der Textilindustrie und der Werften in einer Zeit genannt, in der diese Güter im Ausland zu wesentlich niedrigeren Kosten produziert wurden und ein Wegbrechen dieser Industriezweige in der Bundesrepublik drohte. Die Subventionierung in Form von Investitionshilfen oder steuerlichen Vergünstigungen dient in solchen Fällen der Verlangsamung des wirtschaftlichen Wandels und des zu raschen Abbaus der Arbeitsplätze. Solche verfassungsrechtlich zulässigen Subventionen stoßen freilich, auch unter dem Gesichtspunkt des Sozialstaates, an Grenzen, wenn sie zur Bindung erheblicher öffentlicher Mittel im Interesse der Erhaltung von Wirtschaftszweigen führen und eine Verkrustung der Verhältnisse zur Folge haben, die auf längere Sicht die Erfüllung neuer sozialstaatlicher Aufgaben verhindert. Die bis heute fortge- 
führte Förderung der Werftindustrie ist deshalb zunehmend fragwürdiger geworden. Erst recht problematisch erscheint unter diesem Gesichtspunkt die seit langem bestehende Subventionierung der deutschen Steinkohleförderung. Eine Tonne aus Australien eingeführter Steinkohle kostet per Hafen DuisburgRuhrort etwa 70 DM gegen 280 DM aus heimischer Produktion. ${ }^{71}$ Es mag politische Gründe dafür geben, sich in der Energieversorgung der Bundesrepublik nicht gänzlich vom Ausland abhängig zu machen, wenngleich die Sicherung der Versorgung sich über die deutsche Steinkohle nicht erreichen läßt. Aus dem Sozialstaatsprinzip, hier dem Argument, Arbeitsplätze zu erhalten, läßt sie sich aber nicht rechtfertigen. Denn dies liefe, weitergedacht, auf eine Beschäftigungsgarantie hinaus, die dieses Prinzip in einer Marktwirtschaft nicht hergibt. Gleiches gilt mutatis mutandis für die Förderung des Agrarsektors durch die Bundesrepublik und die EG.

Subventionen werden auch zur Förderung der Produktinnovation und des Aufbaus neuer, zukunftsträchtiger Wirtschaftszweige vergeben. Sie dienen dann vornehmlich der Förderung des Wachstums der Wirtschaft und der Erzielung positiver Erträge der Unternehmen in der Zukunft. Nun lesen wir bereits im Vierten Buch des „Wohlstands der Nationen“"72:

„Der einzelne vermag ganz offensichtlich aus seiner Kenntnis der örtlichen Verhältnisse weit besser zu beurteilen, als es irgendein Staatsmann oder Gesetzgeber für ihn tun kann, welcher Erwerbszweig im Lande für den Einsatz seines Kapitals geeignet ist und welcher einen Ertrag abwirft, der den höchsten Wertzuwachs verspricht".

An die Stelle der örtlichen Verhältnisse, die Adam Smith anführt, läßt sich heute die Marktkenntnis setzen. Doch sind einzelne Unternehmen, auch Großunternehmen, oft gar nicht in der Lage, die immensen finanziellen Aufwendungen für Innovationen, deren Markterfolg nicht sicher ist, aufzubringen. Wenn deshalb in einem geordneten Verfahren unter Nutzung von außerbehördlichem wirtschaftlichen und technischen Sachverstand solche In- 
novationen gefördert werden, um die Ressourcen zu erhalten, die die Finanzierung des Sozialstaats erst gewährleisten, liegt eine sozialstaatlich zulässige Maßnahme vor. Daß Sparförderung und Förderung der Vermögensbildung breiter Kreise ihre Rechtfertigung im Sozialstaatsprinzip finden, bedarf keiner weiteren Begründung.

Die bei weitem interessanteste Frage im Zusammenhang mit der Subventionierung im bundesrepublikanischen Sozialstaat hat die Vereinigung der beiden deutschen Staaten aufgeworfen. Auf den Punkt gebracht, lautet sie: Verlangt das Sozialstaatsprinzip in Verbindung mit anderen Artikeln, die die Wahrung der Wirtschaftseinheit und gleichwertige Lebensverhältnisse beschwören, eine umfassende Förderung der privaten Wirtschaft der neuen Bundesländer? Soweit feststellbar, hat als erster Karl Maria Hettlage auf der Staatsrechtslehrertagung 1972, also lange vor der Vereinigung, diese Verbindung hergestellt. Aus der Kompetenznorm der Art. 72 Abs. 2 Ziff. 3 GG, heute in Art. 72 Abs. 2 GG konkretisiert $^{73}$, aber, was unser Problem angeht, sachlich nicht geändert, lasse sich ein Auftrag zur Förderung der Einheitlichkeit der Lebensverhältnisse als Bestandteil der Wirtschaftseinheit herleiten. Gemeinsam mit Art. 20 GG, der in die gleiche Richtung ziele, ergebe sich daraus ein (mittelbarer) Verfassungsauftrag für die Annäherung der Verhältnisse. Er werde vor allem durch den Finanz- und Lastenausgleich zugunsten der finanzschwachen Länder (Art. 106, 107 GG), die drei großen Gemeinschaftsaufgaben (Art. 91a GG) und die Finanzhilfen des Bundes gem. Art. 104a GG verwirklicht. Nach der Vereinigung sind die Gedanken Hettlages, bezogen auf die neuen Bundesländer wieder aufgenommen worden. ${ }^{74}$ Dabei hat $\mathrm{Arndt}^{75} \mathrm{zu}$ Recht darauf hingewiesen, daß sich erst aus dem Zusammenwirken von Sozialstaatsprinzip und dem Auftrag zur Wahrung der Einheitlichkeit der Lebensverhältnisse ein Anspruch auf angemessene Förderung ergebe, da jedes für sich genommen nur Minimalstandards gewährleiste. Auch auf der Staatsrechtslehrertagung 1992 zur Finanzverfassung des vereinten Deutschland wurde das Sozialstaatsprinzip, das auf unitarische Finanzverhältnisse dränge, angesprochen, wenngleich es gegenüber den speziellen Fragen in den Hintergrund trat. ${ }^{76}$ 
Wendet man sich nach dieser Einstimmung dem Text zu, fällt zunächst auf, daß einige einschlägige Bestimmungen des GG (Art. 72 Abs. 2; 106 Abs. 3 Nr. 2 GG) von der Wahrung der Wirtschaftseinheit oder der Wahrung einheitlicher Lebensverhältnisse sprechen. Daß damit aber auch ein aktives Schaffen einbezogen ist, entspricht herrschender Interpretation. ${ }^{77} \mathrm{Zudem}$ ist in der Verfassung auch vom Ausgleich unterschiedlicher Wirtschaftskraft in der Bundesrepublik (Art. 104a Abs. 4 GG) und von der Herstellung gleichwertiger Lebensverhältnisse die Rede (Art. 72 Abs. 2 GG). Ferdinand Kirchhof geht deshalb ohne weiteres von einer „Pflicht zur Herstellung einheitlicher Lebensverhältnisse im Bundesgebiet" aus. ${ }^{78}$ Sie sei textlich zwar nur für einzelne Aspekte im GG aufgeführt, bestimme jedoch als allgemeiner, ungeschriebener Verfassungssatz das gesamte Finanzgeschehen. Wenn das so ist, kann auch die Kompetenznorm des Art. 72 Abs. 2 GG im Gesamtzusammenhang der Verfassung, des Sozialstaatsprinzips, der Regularien für den vertikalen und horizontalen Finanzausgleich (Art. 106 Abs. 3, 107 Abs. 1 Satz 4 und Abs. 2 GG), der Bundesergänzungszuweisungen (Art. 107 Abs. 2 Satz 3 GG) und Finanzhilfen (Art. 104a Abs. 4 GG) und der mitfinanzierten Gemeinschaftsaufgaben (Art. 91a Abs. 1 GG) nur als Rechtspflicht des Bundes zur Herstellung annähernd gleicher Lebensverhältnisse im Bundesgebiet verstanden werden. ${ }^{79} \mathrm{Daß}$ dies angesichts des noch sehr niedrigen Pro-Kopf-Bruttoinlandsprodukts in den neuen Bundesländern im Vergleich zu den alten ${ }^{80}$ nur in längeren Zeiträumen verwirklicht werden kann, versteht sich von selbst. Die hier konstatierte Pflicht ist zunächst einmal nur eine Förderpflicht gegenüber den neuen Bundesländern. Die Mittel kommen aber, soweit es sich um allgemeine Finanzzuweisungen handelt, auch der Wirtschaft zugute, weil sie den Spielraum der Länder, Wirtschaftsförderung durch Subventionen zu betreiben, erweitern, soweit sie nicht, z. B. bei Gemeinschaftsaufgaben zur Verbesserung der regionalen Wirtschaftsstruktur, unmittelbar die Wirtschaftskraft steigern sollen.

Prozessuale Möglichkeiten, die eben beschriebenen Rechtspflichten durchzusetzen, bestehen freilich nicht für einzelne Unternehmen oder Wirtschaftsverbände, wohl aber für die sich benachtei- 
ligt fühlenden neuen Bundesländer. Sie können im Wege der abstrakten Normenkontrolle überprüfen lassen, ob das jeweilige Finanzausgleichsgesetz mit dem Grundgesetz übereinstimmt ${ }^{81}$. In solchen Verfahren kann das Bundesverfassungsgericht auch überprüfen, inwieweit der Bund überhaupt zum Ausgleich verpflichtet ist. Werden verfassungsgemäße einfach-gesetzliche Normen zugunsten der neuen Bundesländer nicht oder unvollkommen angewendet, ist der Bund-Länder-Streit das geeignete Mittel. ${ }^{82}$

Ausgeklammert wurden die Besonderheiten des Art. 7 des Einigungsvertrages, die Ende 1994 ausliefen, die Maßnahmeprogramme zur Wirtschaftsförderung gem. Art. 28 des Einigungsvertrages, der Fonds „Deutsche Einheit"“83 und die Treuhandanstalt mit Ihren Nachfolgeorganisationen, denen ebenfalls erhebliche Mittel zur Strukturanpassung der Wirtschaft und Sanierung wettbewerbsfähiger Unternehmen in den neuen Ländern ( $\$ 2$ Abs. 6 Treuhandgesetz) zur Verfügung gestellt wurden. Es kam darauf an, den verfassungsrechtlichen „Normalzustand“ aufzuzeigen, der für die Zukunft erhebliche Bedeutung haben wird.

Das Bild wäre aber nicht vollständig, wenn nicht ein Hinweis auf die Subventionsregelung der EG erfolgte. Art. 92 des EG-Vertrages enthält eine im Vergleich zum deutschen Recht einschränkende Regelung. Beihilfen an Unternehmen oder Produktionszweige, die den Wettbewerb verfälschen und den Handel zwischen den Mitgliedstaaten beeinträchtigen, sind danach grundsätzlich verboten. Art. 93 EGV sieht eine Kontrolle der Fördermaßnahmen durch die EG vor. Bestimmte Beihilfen, zu denen auch solche gehören, die der Wirtschaft der durch die Teilung Deutschlands betroffenen Gebiete gewährt werden, sind de jure mit dem Gemeinschaftsrecht vereinbar (Art. 92 Abs. 2 EGV); andere Subventionsmaßnahmen zur Förderung der Entwicklung einzelner Wirtschaftszweige, Wirtschaftsgebiete oder Regionen können als vereinbar angesehen werden (Art. 92 Abs. 3 EGV).

Da das EG-Recht nur Beihilfen ${ }^{84}$ an Unternehmen oder Produktionszweige nennt, fallen die eben diskutierten Hilfen im vertikalen Bund-Länder-Verhältnis bzw. im horizontalen Verhältnis 
der Länder untereinander von vornherein nicht unter das EG-Beihilfeverbot. Ein Konflikt zwischen sozialstaatlich gebotenem und EG-Recht ${ }^{85}$ kann also hier nicht entstehen. Das ist aber nur eine vordergründige Argumentation. Denn soweit die neuen Bundesländer die ihnen zugeteilten Mittel ihrerseits zur Wirtschaftsförderung benutzen oder Beihilfen von den Nachfolgeorganisationen der Treuhandanstalt oder direkt vom Bund gewährt werden, gerät man wieder in den Anwendungsbereich des Art. 92 EGV. Die de jure-Ausnahme für durch die Teilung Deutschlands betroffene Gebiete greift nicht ein; denn die Unternehmen und Produktionszweige der ehemaligen DDR sind nicht wegen der Teilung, sondern durch ihr Gegenteil, die Aufhebung der Teilung benachteiligt. ${ }^{86}$ Es ist mithin auf die in das Ermessen des Rates oder der Kommission gestellte Ausnahmeregelung zur sektoralen und regionalen Strukturförderung zu rekurrieren. Hier scheint freilich Einigkeit darüber zu bestehen, daß sozialstaatlich imprägnierte Fördermaßnahmen, etwa zur Bekämpfung der Arbeitslosigkeit, bei ihrer Subsumtion unter diese Ausnahmemöglichkeit großzügig zu beurteilen sind ${ }^{87}$, ja, daß für eine gewisse Übergangszeit die strengen Vorschriften des Vertrages den Bedürfnissen der Unternehmen in der ehemaligen DDR angepaßt werden müssen. ${ }^{88}$ Gleichwohl geht kein Weg an der Feststellung vorbei, daß angesichts der vom deutschen Recht abweichenden Relevanzkriterien des EG-Vertrages den sozialstaatlich zulässigen Subventionen an die Wirtschaft Grenzen gesetzt werden.

\section{Wirtschaftstätigkeit der öffentlichen Hand}

Ähnlich offen wie gegenüber staatlichen Fördermaßnahmen für Unternehmen, Wirtschaftszweige und Regionen ist das deutsche Recht hinsichtlich der Wirtschaftstätigkeit der öffentlichen Hand. Soweit sie der sog. Daseinsvorsorge ${ }^{89}$ dient, wurde und wird sie auf dem klassischen Feld der sozialstaatlichen Wirksamkeit verortet $^{90}$ und von der rein erwerbswirtschaftlichen Unternehmenstätigkeit des Staates abgegrenzt. Eine Subsidiarität der wirtschaftlichen Betätigung des Staates gegenüber der Privatwirtschaft kennt das deutsche Verfassungsrecht nicht. Die Freiheits- 
rechte setzen nur im Extremfall Schranken. Das Bundesverwaltungsgericht hat einmal formuliert, die Grenze sei erreicht, ,wenn die Wettbewerbsfreiheit des Handels in unerträglichem Maße eingeschränkt wird, eine Auszehrung der Konkurrenz vorliegt oder eine Monopolstellung der öffentlichen Hand besteht ${ }^{\text {"911 }}$. Auf die Gesamtwirtschaft, d. h. die institutionelle Seite der Grundrechte bezogen, wird man kaum mehr als einen signifikanten privaten Sektor und den Ausschluß umfassender staatlicher Planung als verfassungsrechtliches Minimum verlangen dürfen. Angesichts des weiten, wenn auch umstrittenen Bereichs der Daseinsvorsorge, zu dem man die Energie- und Wasserversorgung ${ }^{92}$, den öffentlichen Personennah- und -fernverkehr ${ }^{93}$, Post und Telekommunikation u. a. m. ${ }^{94}$ rechnen kann, und sonstiger öffentlicher Interessen, bildet auch das einfache Recht der Haushaltsordnungen des Bundes ( $\$ 65$ BHO) und der Länder sowie der Gemeindeordnungen keine spürbare Fessel. ${ }^{95}$ Unstreitig ist, daß auch eine als öffentlich-rechtlich zu kennzeichnende Wirtschaftstätigkeit zu keiner Exemtion vom GWB ${ }^{96}$ und $\mathrm{UWG}^{97}$ führt. Im Hinblick auf diese Rechtslage bedarf es sorgfältiger Prüfung, ob zum sozialstaatlichen Selbstverständnis der Bundesrepublik gehörende Wirtschaftstätigkeiten der öffentlichen Hände privatisiert werden sollten. Notorische Finanznöte bieten dafür jedenfalls keine ausreichende Rechtfertigung; auch öffentliche Unternehmen lassen sich rationalisieren. Daß ein mit den privaten Unternehmen in Wettbewerb stehender öffentlicher Sektor heilsame Wirkungen haben kann, zeigt der Kreditbereich. In der jüngsten Diskussion um die Kontoeröffnungspflicht für Sozialhilfeempfänger und vergleichbare Gruppen haben sich die Sparkassen, ihres öffentlichen Auftrages eingedenk, bereit erklärt, solche Konten als „Mindestkonto" zu führen.

Dem Bestreben, den sozialstaatlichen Sektor der Wirtschaftstätigkeit der öffentlichen Hand zu erhalten, setzt, ähnlich wie im Subventionsrecht, der EG-Vertrag Grenzen. Öffentliche Unternehmen, gleichgültig in welcher Rechtsform sie betrieben werden und gleichgültig, in welcher Art sie ihre Tätigkeit gestalten $^{98}$, unterliegen den Wettbewerbsregeln des EG-Vertrages (Art. 90 Abs. 1 EGV). In diesem Zusammenhang hat vor allem das Ver- 
bot der mißbräuchlichen Ausnutzung einer marktbeherrschenden Stellung (Art. 86 EGV) eine eigenständige Bedeutung gewonnen. ${ }^{99}$ Wenn ein öffentliches Unternehmen, wie die Bundesanstalt für Arbeit aufgrund ihres Vermittlungsmonopols unvermeidlich einen Mißbrauch zu Lasten privater Personalberater begeht, da sie nicht in der Lage ist, die Nachfrage auf dem Markt zu befriedigen, ist ein solches Monopol EG-rechtswidrig. ${ }^{100}$ Allerdings sieht der Vertrag eine Ausnahme für Unternehmen vor, die mit Dienstleistungen von allgemeinem wirtschaftlichen Interesse betraut sind (Art. 90 Abs. 2 EGV) . Aber auch diese Legalausnah$\mathrm{me}^{101}$ ist wiederum dadurch eingeschränkt, daß den Vorschriften des Vertrages, insbesondere den Bedingungen des einheitlichen Marktes mit unverfälschtem Wettbewerb, der Vorrang eingeräumt wird, soweit die Erfüllung dieser Dienstleistungen dadurch nicht rechtlich oder tatsächlich verhindert wird. Damit kommen auch die Grundfreiheiten der Waren- und Dienstleistungsfreiheit der Gemeinschaft ins Spiel (Art. 30 ff., 52 ff. EGV). Der Kommission und dem EuGH werden dadurch weite Räume eröffnet, um die öffentliche Wirtschaftstätigkeit einzuschränken.

Im Bereich der Telekommunikation wurde das Monopol der öffentlichen Betreiber von Fernmeldenetzen für Endgeräte beseitigt $^{102}$; ein ausschließlich posteigener Vertrieb von schnurlosen Telefonen würde die Warenverkehrsfreiheit verletzen. ${ }^{103}$ Auf dem Energiesektor wird diskutiert, ob die deutschen Energieversorgungsunternehmen mit Dienstleistungen betraut seien ${ }^{104}$; wäre das nicht der Fall, wären Konzessions- und Demarkationsverträge mit Art. 85 EGV unvereinbar. Da z. B. das französische Monopolunternehmen EdF durch Gesetz mit der Energieversorgung ganz Frankreichs betraut ist, ergäbe sich daraus die merkwürdige Konsequenz, daß gegen EdF nicht, gegen die deutschen Energieversorger aber sehr wohl vorgegangen werden könnte. Daß dies nicht dem Interesse der Gemeinschaft (Art. 92 Abs. 2 EGV) entsprechen würde, hat man inzwischen auch in Brüssel gesehen. ${ }^{105}$ Und schließlich ist streitig, ob die öffentlich-rechtlichen Rundfunk- und Fernsehanstalten überhaupt Dienstleistungen von allgemeinem wirtschaftlichen Interesse erfüllen. ${ }^{106}$ Die Kommission hat sich hier mit ihrem Grünbuch „Fernsehen ohne Gren- 
zen"“107, ebenso wie die Bundesrepublik, den Marktentwicklungen schon längst gebeugt. Aus der grob skizzierten Stoßrichtung des EG-Vertrages und der Politik der Gemeinschaft läßt sich eine deutliche Distanz gegenüber dem sozialstaatlichen deutschen Verfassungsbild ableiten. ${ }^{108}$ In der entscheidenden Frage, der Frage nämlich, welche Aufgaben durch öffentliche Unternehmen und welche durch die Privatwirtschaft wahrgenommen werden sollen, läßt der Vertrag den Mitgliedsstaaten jedoch freie Hand. Denn die Eigentumsordnung ist Sache der Mitgliedsstaaten und nicht der Gemeinschaft (Art. 222 EGV).

\section{Sozialstaatsprinzip und Beschäftigung}

\section{Die verfassungsrechtliche Ebene}

Aus dem Sozialstaatsgebot fließt die staatliche Verpflichtung, Arbeitslosigkeit zu mindern und zu beheben, heißt es in der Entscheidung des Bundesverfassungsgerichts zum Vermittlungsmonopol der Bundesanstalt für Arbeitsvermittlung und Arbeitslosenversicherung. ${ }^{109}$ Deren Tätigkeit dient deshalb einem Gemeinschaftswert, dessen Schutzbedürftigkeit für die industrielle Massengesellschaft allgemein anerkannt und von der jeweiligen sonstigen Gesellschafts- und Wirtschaftspolitik unabhängig ist. Mit den geforderten staatlichen Aktivitäten zur Erreichung der Vollbeschäftigung scheinen wir uns im Zentrum des Sozialstaatsprinzips zu befinden. ${ }^{110}$ Hier gehen die makrosoziale Seite des Prinzips und die mikrosoziale eine enge Verbindung ein. Denn wenn es nicht gelingt, einen hohen Beschäftigungsstand zu erreichen und zu erhalten, wird die Vorsorge für den einzelnen durch die sozialen Sicherungssysteme zunehmend - jedenfalls in bisherigem Umfang - gefährdet.

Zudem verpflichtet Art. 109 Abs. 2 GG alle staatliche Finanzund Wirtschaftspolitik auf das gesamtwirtschaftliche Gleichgewicht, zu dem auch die Sicherung der Vollbeschäftigung gehört ${ }^{111}$, und konkretisiert damit das Sozialstaatsgebot. ${ }^{112}$ Angesichts dessen enttäuscht die fragmentarische Behandlung des Beschäfti- 
gungsproblems auf der verfassungsrechtlichen Ebene. ${ }^{113}$ Streit besteht über den Standort der Vollbeschäftigung im Rahmen des gesamtwirtschaftlichen Gleichgewichts. Während sie Scholz u. a. durch die weiteren Bestandteile relativiert, d. h. wohl eingeschränkt sehen ${ }^{114}$, betont Kittner deren Vorrang. Die drei weiteren Ziele seien demgegenüber lediglich instrumentell und damit im Lichte des Sozialstaatsprinzips nur deshalb zu rechtfertigen, als wirtschaftspolitisch plausibel dargetan werden könne, daß durch die Verfolgung des ganzen Zielbündels auf lange Sicht gerade ein hoher Beschäftigungsstand gesichert werden könne. ${ }^{115}$ Daran ist zutreffend, daß der in $\S 1$ StabilG genannte übergeordnete Gesamtrahmen der „marktwirtschaftlichen Ordnung“ sich in der Verfassung (Art. 109 Abs. 2 GG) nicht findet und deshalb das Sozialstaatsgebot auf dieser Ebene eine besondere Wirkungskraft entfalten könnte. Kittner übersieht aber, daß durch die Verfassung selbst die vornehmlich auf Wachstum orientierte privatwirtschaftliche Betätigung und die auf (relative) Stabilität des Preisniveaus angewiesene Geld- und Vermögensanlage geschützt sind (s. o. I). Er übersieht ferner, daß die Kriterien des gesamtwirtschaftlichen Gleichgewichts nur die Konjunkturpolitik des Staates bestimmen können ${ }^{116}$, hohe Arbeitslosigkeit aber auch andere, nicht konjunkturbedingte Ursachen hat. Schließlich liegt sein bereits zitierter polemischer Hinweis auf Art. 1 GG auf der Linie der Sozialcharten ${ }^{117}$ und Erklärungen der Menschenrech$t^{118}$, die Zielprogramme enthalten, aber keine konkreten Verbindlichkeiten für die Wirtschaftsordung oder Rechtsansprüche einzelner erzeugen können. Das Bundesverfassungsgericht hat in seinen zahlreichen Äußerungen zur Bedeutung der Menschenwürde diese nie mit der Vollbeschäftigung assoziiert. ${ }^{119}$ In der Rechtsprechung der Arbeitsgerichte wird die Menschenwürde nicht mit der Vollbeschäftigung, sondern mit dem bestehenden Arbeitsverhältnis verknüpft. ${ }^{120}$

Wenig hilfreich ist auch die in den letzten Jahren geführte Diskussion um ein bundesverfassungsrechtliches Recht auf Arbeit, das im Wege der Kumulierung der Einzelansprüche zur Vollbeschäftigung führen würde. Es ist unstreitig, daß das GG keinen einklagbaren Anspruch auf einen Arbeitsplatz als ,,soziales Grund- 
recht“" oder „Teilhaberrecht“ gewährt. ${ }^{121}$ Die sich in zahlreichen Verfassungen der Bundesländer findende Verbürgung eines Rechts auf Arbeit ${ }^{122}$ stellt entweder einen Programmsatz dar oder ist bundesverfassungskonform so zu interpretieren. ${ }^{123}$ Die mageren Ergebnisse des verfassungsrechtlichen Diskurses haben ihren Grund letztlich darin, daß die marktwirtschaftliche, verfassungsrechtlich ohne Zweifel zulässige Ordnung der Bundesrepublik keine Vollbeschäftigung garantieren kann, ebenso wie sie den Unternehmen nicht den Absatz ihrer Produkte garantiert. ${ }^{124}$

\section{2. Ökonomische Maßnahmen und Initiativen der Koalitionspartner}

Deshalb wird bevorzugt auf ökonomische Maßnahmen verwie$\operatorname{sen}^{125}$ und die Verkrustung der durch die Tarifvertragsparteien bestimmten Strukturen des Arbeitsmarktes gerügt. ${ }^{126}$ Nach neoklassischer Sicht könnten Ungleichgewichte in Form der Arbeitslosigkeit durch den Reallohnmechanismus, d. h. niedrigere Löhne, ausgeglichen werden. ${ }^{127}$ Die Keynessche Lehre setzt dem entgegen, die Grundannahmen der klassischen Gleichgewichtstheorie träfen auf den Arbeitsmarkt nicht zu. Die Nachfrage nach Arbeit werde nicht durch das Reallohnniveau, sondern durch die Höhe der effektiven Güternachfrage bestimmt. Durch Geld- und Fiskalpolitik sowie Investitionssteuerung des Staates, also durch staatliche Makropolitik, könne chronische Arbeitslosigkeit unterbunden werden. ${ }^{128}$ Beides wird tatsächlich, mit bisher unzureichendem Erfolg, in der Bundesrepublik versucht oder ins Auge gefaßt. Stichworte sind die Flexibilisierung der Tarifverträge, die Einführung einer Bezahlung unter Tarif während der Zeit nach Arbeitsaufnahme, die Niedrigzinspolitik der Bundesbank, Umverteilung durch Steuerprogression und Solidaritätszuschlag, Investitionsförderung und öffentliche Infrastrukturinvestitionen $u$. a. m. Die offene Arbeitslosigkeit von über 4 Mio. erwerbsfähiger Personen in der Bundesrepublik und die durch Arbeitsförderungs- und Arbeitsbeschaffungsmaßnahmen verdeckte von nochmals über 1,5 Mio. dürfte dadurch kaum zu beseitigen sein. 
3. Verpflichtungen des einfachen Gesetzgebers

Der Jurist muß deshalb fragen, ob der einfache Gesetzgeber aufgrund des Sozialstaatsprinzips gehalten ist, die Bedingungen für Vollbeschäftigung zu verbessern und wie er dies bewerkstelligen kann. Dazu einige Hinweise:

In erster Linie geht es darum, die Bedingungen für die Erhaltung bestehender und die Schaffung neuer Arbeitsplätze zu verbessern. Dem stehen auch die Erstarrung der Arbeitmärkte, die durch das deutsche Arbeitsrecht unterstützt wird, und die Vorschriften, die bei Aufnahme und Fortführung unternehmerischer Tätigkeit zu beachten sind, entgegen. Die Reduzierung des Arbeitsrechts auf Interessengegensätze zwischen Arbeitgeber und Arbeitnehmer verkennt den gesamtwirtschaftlichen Systemzusammenhang. So macht es keinen Sinn, Tarifverträge, die häufig an der Situation leistungskräftiger Großunternehmen orientiert sind, für allgemeinverbindlich zu erklären. Zwar sind in der Bundesrepublik per 1.1.1996 von 43.600 eingetragenen Tarifverträgen nur 565 allgemeinverbindlich $^{129}$ und erfassen damit auch die bisher nicht tarifgebundenen Arbeitgeber und Arbeitnehmer ( $\$ 5$ Abs. 4 TVG); diese kleine Zahl betrifft aber einen erheblichen Teil der Beschäftigten. ${ }^{130}$ Nachdem immer mehr Unternehmen - vor allem kleinere und mittlere, die ihre Arbeitsplätze nicht in gleichem Umfang wie Großunternehmen abbauen - aus ihren Arbeitgeberverbänden ausgeschieden sind, und die Löhne auf betrieblicher Ebene aushandeln, ist es kontraproduktiv, das gesetzliche Instrument der Allgemeinverbindlicherklärung aufrechtzuerhalten. Ein weiteres Beispiel: Gem. § 112 Abs. 1 Satz 2 Betriebsverfassungsgesetz haben Unternehmen bei Betriebsänderungen einen Sozialplan aufzustellen. Die Bestimmung wird dahin verstanden, daß z. B. die Verlegung einer Betriebsabteilung innerhalb einer Großstadt in ein 4,3 km vom bisherigen Betriebsort entfernt liegendes Gebäude sozialplanpflichtig sei. ${ }^{131}$ Die darin zum Ausdruck kommende geringe Flexibilität gefährdet die Arbeitsplätze, um die es geht; der Gesetzgeber ist gefordert.

Die vielfältigen Hemmnisse, die Verwaltungsrecht und Verwaltungsverfahren der Aufnahme und Ausübung unternehmeri- 
scher Tätigkeit in Deutschland entgegensetzen, haben sich bereits international $\mathrm{zu}$ einem schlechten Markenzeichen entwickelt. Thad Perry, Leiter der deutschen Niederlassung der Andersen-Consulting bemerkte dazu kürzlich ${ }^{132}$, in Deutschland gebe es für die Unternehmensgründung nur Einschränkungen und Auflagen; die Verwaltung sei katastrophal, zwar sehr präzise, aber absolut ineffektiv. Der Rückgang des Saldos von Unternehmensneugründungen und -liquidationen von knapp 233.000 im Jahre 1991 auf 121.000 im Jahre $1994^{133}$ sollte zu denken geben.

Als wenig hilfreich dürften sich dagegen protektionistische Maßnahmen, wie das soeben beschlossene Entsendegesetz erweisen. Soweit es aus den EU-Ländern kommende Unternehmen und Arbeitnehmer betrifft, bestehen Konflikte mit der EGrechtlichen Niederlassungs- und Dienstleistungsfreiheit (Art 52, $59 \mathrm{EGV})^{134}$; ist das nicht der Fall, reicht das deutsche Ausländerrecht, das Aufenthalts- und Arbeitsgenehmigungen voraussetzt, aus, um die berechtigte Bevorzugung inländischer Arbeitsloser durchzusetzen. Im übrigen führen protektionistische Regelungen, soweit die Arbeit nicht platzgebunden ist, zu Ausweichstrategien der Unternehmen und gefährden auf längere Sicht inländische Arbeitsplätze.

Neben legislative Aktivitäten, die der Erhaltung und Schaffung neuer Arbeitsplätze dienen, müssen Gesetzesänderungen treten, die dazu ermuntern, aus dem Arbeitsleben auszuscheiden. Ich nenne nur die Verkrustung des Beamtenrechts. Beamte können auf Antrag mit dem 62., künftig 63. Lebensjahr aus dem Dienst ausscheiden, früher aber nicht. ${ }^{135}$ Aus der Generation der Erben mag es eine ganze Reihe geben, die den erforderlichen Pensionsabschlag in Kauf nehmen und früher ausscheiden würden; wahrscheinlich auch viele aus der großen Gruppe, die heute über den Weg der Dienstunfähigkeit alle Vorteile miteinander zu verbinden sucht. Die patriarchalische, auf dem Alimentationsprinzip beruhende Grundeinstellung des Beamtenrechts ist längst überholt. 
Es gibt in beiden Teilen Deutschlands mehr Arbeit als Arbeitsplätze. Beleg dafür ist die umfangreiche Schattenwirtschaft vor allem auf dem Sektor der Dienst- und Werkverträge, die zum Sozialprodukt beiträgt und den regulären Arbeitsmarkt entlastet. Das Recht verabscheut die Schwarzarbeit: Die Verträge sind nichtig ( $\$ 134$ BGB), die Tätigkeit als solche wegen des durch Rechtsbruch erzielten Vorsprungs im Wettbewerb unlauter (§ 1 UWG). Arbeits-, Sozial- und Finanzbehörden im Verbund mit Kammern, Innungen und Gewerkschaften kontrollieren den Neben- oder Haupterwerb des Schwarzarbeiters mit Argusaugen und bedienen sich dazu auch der Polizei. Viele scheuen deshalb das Risiko und werden für den legalen Arbeitsmarkt immer untauglicher. Unter arbeitsmarktpolitschem und sozialstaatlichem Aspekt ist aber demjenigen, der seine Arbeitskraft selbst vermarktet, dies zu erleichtern und nicht zu erschweren. Vorschläge, wie eine Legalisierung in einer diesen Arbeitssektor fördernden und ihn nicht pönalisierenden Weise geschehen könnte, liegen seit langem vor. Sie reichen von der steuerlichen Anerkennung der Privathaushalte als Arbeitgeber ${ }^{136}$ über die Öffnung der Sozialversicherung für Eigen-Unternehmer zum halben Beitragssatz ${ }^{137}$ bis zur Erhöhung des nicht anrechenbaren Zuerwerbs in der Sozialfürsorge und Arbeitslosenhilfe.

Schließlich noch ein Wort zu der von Kittner aufgeworfenen Frage, ob verdeckte Arbeitslosigkeit nicht sozialstaatlicher sei als offene. ${ }^{138}$ Konkret gesagt, sind jedenfalls die öffentlichen Hände verpflichtet, die Rationalisierung zu verlangsamen, um Arbeitsplätze zu erhalten oder neu zu schaffen? Die Frage ist zu verneinen. Ebenso wie in der Privatwirtschaft falsche Faktorkombinationen, die unwirtschaftliche Arbeit begünstigen und Kapitaleinsatz verlangsamen, zu einer Pauperisierung der Gesamtheit der Erwerbstätigen führen, ist auch die öffentliche Hand verpflichtet, die ihr von der Gesamtheit der Steuerzahler zur Verfügung gestellten Mittel effizient, d. h. im Sinne einer Intensivierung ihres „Kapitals“, das auf Modernisierung drängt, einzusetzen.

Ich breche hier ab und füge ein ceterum censeo hinzu: 
4. Zum Wert bezahlter Arbeit und selbständiger Erwerbstätigkeit

In den Industriestaaten westlicher und sogen. realsozialistischer Prägung wurde und wird Arbeitslosigkeit als ein widernatürlicher Zustand betrachtet mit den bekannten Auswirkungen auf die Selbstachtung des Arbeitslosen. ${ }^{139}$ Diese Einstellung ist hypertroph; sie vernachlässigt den Wert der karitativen Tätigkeit und der Hausfrauentätigkeit, um nur die wichtigsten Felder sinnvollen und das Sozialprodukt erhöhenden menschlichen Tuns zu nennen. Sie wurzelt aber tief in der Kulturgeschichte des Westens, so daß die notwendige Bewußtseinsänderung nur schwer zu erreichen sein wird. Max Weber hat in seiner protestantischen Ethik die Linie zum asketischen Protestantismus der Calvinisten und Puritaner gezogen, die für den „Geist“ des Kapitalismus von Bedeutung seien. ${ }^{140}$ Auf die Initialzündung, die diese Vorstellungen für den Übergang von traditionalistischen, an konstanten Minimaleinkünften orientierten Arbeitseinkommen zu arbeitsteiligen und marktwirtschaftlichen bewirkten, ist auch andernorts hingewiesen worden. ${ }^{141}$ Gar nicht weit davon entfernt ist Karl Marx, wenn er die Arbeit zur Existenzbedingung des Menschen, in der sich seine Gattungsnatur verwirkliche, übersteigert. ${ }^{142}$ Unter der Herrschaft der Arbeiterklasse hat diese, wohl falsch verstandene Lehre ${ }^{143}$, zum gesellschaftlichen Zwang der Berufstätigkeit aller Erwerbsfähigen geführt. Ist auch ohne bezahlte Arbeit ein ausreichender Lebensunterhalt gesichert, können andere Werte legitimerweise Vorrang gewinnen; selbst die Muße der Kontemplation, für Aristoteles der höhere Wert gegenüber der Arbeit ${ }^{144}$, verdient kein moralisches Unwerturteil.

\section{Sozialstaatsprinzip und Privatrechtsordnung}

\section{Grundfragen}

Die Realität des Wirtschaftens wird durch Verträge bestimmt; ihr Recht ist deshalb Gegenstand der Betrachtung. Die Verbindung zwischen dem Sozialstaatsgebot als einem auch die Wirtschaftsordnung prägenden Prinzip und der Privatrechtsordnung läßt sich nur herstellen, wenn man vom einzelnen Vertrag abstrahiert, ihn 
in quantifizierbaren Größen untergehen läßt und als Bestandteil einer dezentral geplanten Wirtschaft erfaßt ${ }^{145}$, ihn mithin als Institution versteht.

Aus diesem Grunde ist es methodisch unzulässig, das Sozialstaatsprinzip generell in den individuellen Vertrag als Pflicht zu sozialem Verhalten hineinzulesen. Schon Ballerstedt hat darauf hingewiesen, daß durch eine solche unmittelbare Drittwirkung des Prinzips die Privatrechtsordnung gesprengt würde. ${ }^{146}$ Noch deutlicher hat Charles Fried in seinem Buch „Contract as Promise“, 1981, den Vertrag auf sein theoretisches Skelett zurückgeführt:

It is unfair (and in the end counterproductive) to force particular persons, who are making their private arrangements against the background of conditions they did not create, to bear the burden of remedying these conditions. The position of a just social minimum should be society's general responsibility, not the responsibility of individual economic actors, except as they pay taxes to fund that minimum. ${ }^{147}$

Die Richtigkeitsgewähr des Vertrages ist also nicht deshalb in Frage zu stellen, weil in bestimmten Vertragsverhältnissen (im Arbeitsrecht oder im Verbraucherschutzrecht) die Privatautonomie nicht den sozialen Gesetzgeber zu ersetzen vermag. ${ }^{148}$

Der deutsche Gesetzgeber hat bekanntlich auf weiten Feldern des Vertragsrechts, auf denen er eine strukturelle Unterlegenheit des Vertragspartners als gegeben ansah, die Vertragskonditionen bestimmt und die Funktion des Vertrages eingeschränkt. Es handelt sich um Konstellationen, bei denen ökonomisch-sozial begründete Macht in der rechtlichen Bewertung, jedenfalls nach der Auffassung des Gesetzgebers, hoheitlicher Gewalt gleichgestellt werden kann. ${ }^{149}$ Sie sind deshalb genuiner Ausdruck des Sozialstaatsprinzips. Die Entwicklung beginnt lange vor dem Inkrafttreten des GG mit dem preußischen Regulativ über die Beschäftigung jugendlicher Arbeiter in Fabriken vom 9. März $1839^{150}$ und reicht über das Individualarbeitsrecht, das seit 1917 einsetzende 
Mieterschutzrecht ${ }^{151}$ und das Abzahlungsgesetz von 1894 hinaus bis zu den heutigen, wesentlich durch EG-Recht beeinflußten, Verbraucherschutzgesetzen. ${ }^{152}$

Davon zu unterscheiden ist die Gruppe derjenigen Gesetze, die Marktfreiheit erst herstellen oder Marktmacht binden sollen. Sie gehören gedanklich in das Kapitel Sozialstaatsprinzip und Markt (s. o. I), sollen wegen ihrer privatrechtlicher Relevanz aber nochmals angesprochen werden. So unterliegen Monopolbetriebe, wie die Eisenbahnen und die Energieversorgungsunternehmen einem gesetzlichen Kontrahierungszwang, Tarif- und Betriebszwang ( $\$ 6$ EVO, $§ 6$ EnergiewirtschaftsG), sind Kartellverträge gem. § $1 \mathrm{GWB}$ unwirksam. Gegen überhöhte Preise, die unter Ausnutzung wirtschaftlicher Machtstellung gefordert werden und den Wettbewerb beschränken, kann nicht nur die Kartellbehörde vorgehen ( $\$ 22$ Abs. 4 Nr. 2 GWB); sie sind auch nach $\S 4$ WirtschaftsstrafG i. V. m. § 134 BGB unwirksam oder auf die zulässige Höhe zurückzuführen. ${ }^{153}$ Der Grund für diese Regelungen liegt nicht in der Unterlegenheit des Vertragspartners, sondern in der Marktbeeinträchtigung durch den Handelnden; sie sind Ausdruck sozialstaatlicher Marktsteuerung.

Das überkommene Privatrecht sieht darüber hinaus in Form des unbestimmten Prinzips der Sittenwidrigkeit (§ 138 BGB) und der Verhaltenspflicht nach Treu und Glauben ( $\$ 242$ BGB) auch selbst Grenzen der Vertragsordnung vor. Hier ist die mittelbare Einbruchstelle des Sozialstaatsprinzips in das Privatrecht. Das Prinzip verdrängt und ersetzt aber nicht diese Rechtsnormen ${ }^{154}$ und das durch Rechtsprechung und Lehre entwickelte ausdifferenzierte dogmatische System. Es bestimmt jedoch als Bestandteil der ,verfassungsmäßigen Ordnung“ die Auslegung in Fällen struktureller Störungen der Vertragsparität. ${ }^{155}$ In der bekannten Entscheidung des Bundesverfassungsgerichts zu Bürgschaften junger Erwachsener, die durch hohe Verpflichtungen für Eltern oder Partner in eine ausweglose Überschuldung gerieten ${ }^{156}$, hat deshalb das Gericht festgestellt, das geltende Vertragsrecht genüge den Anforderungen, die in solchen Fällen an die Privatrechtsordnung zu stellen seien, m. a. W. der IX. Zivilsenat des BGH ha- 
be nur das geltende Recht falsch gewürdigt. ${ }^{157} \mathrm{Daß}$ das Sozialstaatsprinzip sich auf die vertragliche Gestaltungsfreiheit auswirkt und damit dem Vertrag als Institution eine nicht nur freiheitssichernde Funktion zuweist, entspricht im übrigen der ständigen Rechtsprechung des Verfassungsgerichts seit früher Zeit. ${ }^{158}$

\section{Gefahren}

Die Imprägnierung des Privatrechts durch das verfassungsrechtliche Sozialstaatsprinzip, wie sie in dieser Rechtsprechung deutlich wird, birgt freilich auch Gefahren für die Wirtschaftsordnung in sich. Eine sozialstaatlich gerechtfertigte Mieterschutzgesetzgebung kann dazu führen, daß sich die Investoren aus dem Mietwohnungsbau zurückziehen und der Staat erhebliche Mittel aufwenden muß, um das fehlende private Engagement zu ersetzen oder durch ausgleichende Incentives erneut zu gewinnen. Ein überzogener Arbeitnehmerschutz wirkt sich hinderlich auf die Entstehung neuer Arbeitsplätze und die Ansiedlung ausländischer Unternehmen in der Bundesrepublik aus. Das steigende Ungleichgewicht deutscher Direktinvestitionen im Ausland gegenüber ausländischen Direktinvestitionen im Inland ${ }^{159}$ ist ein warnendes Signal. Es bedarf deshalb einer sorgfältigen Abwägung zwischen sozialstaatlich Erwünschtem und einer gesamtwirtschaftlich unerwünschten zu starken Behinderung der Marktkräfte. Gerichte sind dazu wegen ihrer Ausrichtung auf den Einzelfall regelmäßig nicht in der Lage; den Juristen insgesamt fehlt häufig der wirtschaftsrechtliche und erst recht der ökonomische Blick. Eine dem deeppocket-Ansatz folgende Verbraucherschutzgesetzgebung zu Produkthaftungs- und Beratungspflichten übersieht deshalb nicht selten, daß der damit verbundene Kosteneffekt häufig nur zu Umverteilungen auf der Verbraucherseite führt.

Die betonte Einwirkung des Sozialstaatsprinzips auf die Vertragsordnung kann schließlich zur Erosion der zivilrechtlichen Argumentation mit negativen Folgen für die Rechtssicherheit, ja zur Kadijustiz führen. Ein neues Urteil des LG Göttingen ${ }^{160}$ mag dafür ein Beleg sein: Das Gericht hatte über die rechtliche Wirksamkeit ei- 
ner Vermögensanlage in Form eines Pensionssparplans zu entscheiden und befand, daß der Prospekt, der den Anleger über diesen Sparplan informieren sollte, zu kompliziert für Personen mit „geringerer wirtschaftlicher und rechtlicher Erfahrung“ sei. Selbst vorgebildete Leser, wie die Mitglieder der Kammer, hätten mindestens einen Tag gebraucht, um die wesentlichen Einzelheiten zu erfassen. So weit so gut. Nach der Rechtsprechung des BGH ${ }^{161}$ darf ein Vermögensanlageprospekt an das Niveau eines Lesers gewisse Anforderungen stellen, sie dürfen aber nicht überspannt werden. Der BGH setzt einen aufmerksamen Leser voraus, der zwar eine Bilanz zu lesen versteht, aber über kein überdurchschnittliches Fachwissen verfügt. Sind diese Anforderungen nicht erfüllt, kommt es zur sog. Prospekthaftung, bei der Verschulden desjenigen, der den Prospekt erlassen hat und Mitverschulden des Adressaten zu berücksichtigen sind. Es kann nach Lage des Falles auch eine arglistige Täuschung vorliegen, die den Vertrag anfechtbar macht. Das alles hat das Gericht aber nicht geprüft. Es leitet vielmehr aus dem zu komplizierten Prospekt eine strukturelle Unterlegenheit des Prospektadressaten und daraus unter ausdrücklichem Hinweis auf das Sozialstaatsprinzip eine Nichtigkeit des Vertrages gem. $\S 138$ BGB ab. Eine solche Entscheidung, die nach krauser Gedankenführung mit dem Hammer zuschlägt, ließe man einem Jurastudenten nicht durchgehen. Wie Otto von Gierke in seinem berühmten Wiener Vortrag ${ }^{162}$ betont hat, besitzt das Privatrecht zwar durchaus eine soziale Aufgabe; dort heißt es aber auch ,die Verstaatlichung des Privatrechts im Sinne des Sozialismus (heute zu lesen: eines rigiden Sozialstaatsprinzips) bedeutet die Unfreiheit und die Barbarei.“

\section{Zusammenfassung}

1. Die Wirtschaftsordnung der Bundesrepublik wird durch das Sozialstaatsprizip zunächst in ihren äußersten Grenzen bestimmt. Eine laissez-faire-Wirtschaft ist ebenso verfassungsrechtlich unzulässig wie eine Wirtschaftsordnung, die privater wirtschaftlicher Betätigung keinen Raum läßt und sie durch staatliche Planung oder Wirtschaftstätigkeit der öffentlichen Hände in den Hin- 
tergrund drängt. Die verfassungsrechtlich garantierte freie wirtschaftliche Betätigung setzt allen staatlichen Aktivitäten, die sie zu ersticken drohen, verfassungsrechtliche Grenzen.

2. Die durch einfach-gesetzliches Recht und durch den EG-Vertrag etablierte Wettbewerbsordnung darf nicht als die Erfüllung des Sozialstaatsprinzips verstanden werden. Insbesondere ist die Begrenzung staatlicher Einflußnahme auf marktkonforme Maßnahmen mit dem Sozialstaatsprinzip nicht kompatibel.

3. Es sind deshalb auch wettbewerbswidrige staatliche Maßnahmen, wie Subventionen, zulässig, zur Herstellung einheitlicher Lebensverhältnisse in der Bundesrepublik nach der Vereinigung sogar geboten.

4. Die Wirtschaftstätigkeit der öffentlichen Hände, insbesondere auf dem Sektor der Daseinsvorsorge, läßt sich weder unter Berufung auf das GG noch auf den EG-Vertrag einschränken. Allerdings sind auch die Unternehmen der öffentlichen Hand, ohne Rücksicht auf ihre Rechtsform, den Wettbewerbsregeln unterworfen und auf die Grundfreiheiten des EG-Vertrages verpflichtet.

5. Die verfassungsrechtliche Verpflichtung auf einen möglichst hohen Beschäftigungsstand zwingt den einfachen Gesetzgeber und die staatliche Wirtschaftspolitik, die Bedingungen für die Erhaltung bestehender und die Schaffung neuer Arbeitsplätze zu verbessern. Hier bestehen z. Zt. erhebliche Defizite; vor allem werden die Auswirkungen von Einzelmaßnahmen, die vordergründig arbeitsplatzerhaltend wirken, auf das Beschäftigungsniveau insgesamt verkannt. An der Ermunterung, ohne Belastung öffentlicher Haushalte und der Solidarkassen aus dem Arbeitsprozeß auszuscheiden, fehlt es; die erforderliche Bewußtseinsänderung wird tabuisiert.

6. Die Privatrechtsordnung läßt sich nicht durch eine unmittelbar geltende Verpflichtung zu sozialem Verhalten, abgeleitet aus dem Sozialstaatsprinzip, demontieren. Das Prinzip beschränkt aber die Funktion des Vertragsrechts, Ungleichgewichte durch 
Willenseinigung zu rechtfertigen, wenn ökonomisch oder sozial begründete Macht das freiheitliche Prinzip des Vertrages konterkariert. 


\section{Anmerkungen}

1 A. a. O. S. 5 f., 9, 14; ebenso Recktenwald in: Einleitung zu Adam Smith, Der Wohlstand der Nationen, Neudruck 1974, S. XXXV ff.

2 A. a. O. S. 10.

3 A. a. O. S. 20. Keynes weist vor allem auf Cairnes und Alfred Marshall hin; siehe auch Dräger in Ritter/Gründer, (Hrsg.), Historisches Wörterbuch der Philosophie Bd. 5, Basel, 1980, S. 256, 270.

4 A. a. O. S. $30 \mathrm{ff}$.

5 Auf die verfassungsrechtliche Bedeutung des Kostenmoments weist Stern, Das Staatsrecht der Bundesrepublik Deutschland, Bd. I, 2. Aufl. 1984, S. 908 ff., 911 hin.

6 A. a. O. (FN 5), S. 914.

7 Herzog in: Maunz/Dürig/Herzog/Scholz, GG, Art. 20 VIII, Rdnr. 1.

8 Rechtsstaat - Sozialstaat - Staat, Hamburger Öffentlich-rechtliche Nebenstunden, Bd. 29, 1975, S. 25.

9 In: Bettermann/Nipperdey/Scheuner (Hrsg.), Die Grundrechte, Bd. III, 1, 1958 S. 1, $50 \mathrm{f}$.

10 So von Zacher, in: Isensee/Kirchhof (Hrsg.), Handbuch des Staatsrechts, Bd. I, 1987 S. 1059 ff.; 1078 ff.

11 So von Stern a. a. O. (FN 5), S. $893 \mathrm{ff}$.

12 Stern a. a. O. (FN 5), S. 914.

13 BVerfGE 22, 180, 204 (Mithilfe privater Wohlfahrtsorganisationen); vgl. auch BVerfGE 59, 231, 263 unter Hinweis auf die Begrenzung des Sozialstaatsprinzips durch das Demokratieprinzip.

14 Dazu näher Luhmann, Das Recht der Gesellschaft, 1993, S. 195 ff.

15 Zacher a. a. O. (FN 10), S. 1084.

16 Vgl. hierzu Stern a. a. O. (FN 5) und Böckenförde, Der Staat, Bd. 15 (1976), S. 470.

17 In dieser Richtung aber AK z. GG - Kittner, Art. 20, IV, Rdnr. 55.

18 A. a. O. (FN 9), S. 21.

19 BVerfGE 82, 80.

20 Kittner a. a. O. (FN 17), Rdnr. 55, will die positive Justitiabilität nur im Regelfall ausschließen, unter Hinweis auf BVerfGE 1, 97, 105; 50, 57, 108.

21 BVerGE 29, $402 \mathrm{ff}$.

22 BVerfGE 26, 7.

23 BVerfGE 13, 259.

24 BVerfGE 12, 354, $363 \mathrm{ff}$.

25 BVerfGE 8, 274, 329; dazu L. Raiser, JZ 1958, 1 ff. 
26 BVerfGE 14, 263, 286.

27 BVerfGE 87, 153, $170 \mathrm{f}$.

28 A. a. O. (FN 10), S. 1088; in der Sache ebenso Stern a. a. O. (FN 5), S. 902.

29 Ähnlich Rinck/Schwark, Wirtschaftsrecht, 6. Aufl., S. 17.

30 So der Wirtschaftsordnung sbegriff Euckens, Grundsätze der Wirtschaftspolitik, 1952, S. 23

31 So bezeichnet Ballerstedt die rechtliche Ordnung der Wirtschaftsgemeinschaft als Wirtschaftsverfassung, a. a. O. (FN 9), S. 1, 17.

32 Dazu vgl. insbesondere Raisch, Zum Nutzen der überkommenen Auslegungskanones für die praktische Rechtsanwendung, 1988; ders., Juristische Methoden, 1995, S. $133 \mathrm{ff}$.

33 Zacher a. a. O. (FN 10), S. 1061; Stern a. a. O. (FN 5), S. 923.

34 Papier, Grundgesetz und Wirtschaftsordnung, in: Benda/Maihofer/Vogel (Hrsg.), Handbuch des Verfassungsrechts, Bd. 1, 2. Aufl. 1995, $\S 18$ Rdnr. 34 ff., 75 ff.; R. Schmidt, Öffentliches Wirtschaftsrecht, Allgemeiner Teil, 1980, § 4 I 2, S. $117 \mathrm{ff}$.

35 Papier, a. a. O. (FN 34), Rdnr. 62 ff.; Löwer in: von Münch/Kunig (Hrsg.), GG-Kommentar, Bd. 1, 4. Aufl. 1992, Art. 9, Rdnr. 28; Schmidt a. a. O. (FN 34), S. $147 \mathrm{ff}$.

36 Bender in: G. Briefs (Hrsg.), Laissez-faire-Pluralismus, 1966, S. 347.

37 Stern a. a. O. (FN 5), S. 923.

38 Zitiert nach Rinck, Wirtschaftsrecht, 5. Aufl. 1977 (Eingangsmotto); in der 6. Aufl. von mir gestrichen.

39 Stein, Staatsrecht, 8. Aufl., § 6 IV; E. Denninger in: Görlitz, Handlexikon zur Rechtswissenschaft, 1924, 424 ff.; kritisch Kloepfer, Gleichheit als Verfassungsfrage, 1980, S. 43 f.; differenzierend Stern a. a. O. (FN 5), S. 929 f. m. w. N.; vgl. auch BVerfGE 45, 376, 385 ff.; 55, 100, $111 \mathrm{f}$.

40 A. a. O. (FN 17), Art. 20, IV, Rdnr. 41, 56.

41 S. vor allem die Beiträge in: Böckenförde (Hrsg.), Staat und Gesellschaft 1976; Ballerstedt a. a. O. (FN 9), S. 47; Mestmäcker, Markt Recht - Wirtschaftsverfassung in: Recht und ökonomisches Gesetz, 2. Aufl. 1984, S. 15 ff.; kritisch Ehmke, Wirtschaft und Verfassung, 1961, S. 55 u. passim; gänzlich ablehnend Abendroth, FS Bergstraesser, 1954, S. 279 ff., 288, der die gesamte Gesellschafts- und Wirtschaftsordnung durch das GG zur Disposition der demokratischen Willensbildung gestellt angesehen hat.

42 Wirtschaftslenkung und Marktwirtschaft, 1947, S. 88.

43 Dazu Müller-Volbehr, JZ 1982, 132, $133 \mathrm{f}$.

44 BVerfGE 4, 18 - Investitionshilfeurteil; wiederaufgenommen im Mit- 
bestimmungsurteil, BVerfGE 50, 290, 338.

45 VVDStR1 10 (1952), S. 124, 147; später z. T. a. M. ders. in: Die soziale Ordnung des Grundgesetzes, 1975, S. $100 \mathrm{ff}$.

46 Dazu Müller-Volbehr, JZ 1982, 132, $133 \mathrm{f}$.

47 In dieser Richtung aber Häberle, ZRP 1993, 383 ff.

48 Statt aller Kittner a. a. O. (FN 17), Art. 20, IV, Rdnr. 71.

49 Näheres bei Rinck/Schwark, Wirtschaftsrecht, 6. Aufl., S. 52 ff.

50 Denn die weiteren Bestandteile gesamtwirtschaftlichen Gleichgewichts können in gleicher Weise Gegenstand der Wirtschaftspolitik ganz unfreiheitlicher planwirtschaftlicher Systeme wie auch eines laissez-faire-Systems sein, das Wachstum und außenwirtschaftliches Gleichgewicht durch staatliche Zurückhaltung - mit Ausnahme einer Preisstabilität sichernden Geldmengensteuerung - zu erreichen sucht.

51 A. a. O. (FN 48), Rdnr. 71.

52 BVerfGE 21, 245, 251.

53 Vgl. nur Denninger in: Görlitz (Hrsg.), Handlexikon zur Rechtswissenschaft, 1974, $424 \mathrm{ff}$.

54 So Stern a. a. O. (FN 6), S. 910; bedenklich auch BVerfGE 59, 231, 263.

55 Vgl. nur Stern a. a. O. (FN 6), S. 929 m. w. N.; a. A. z. B. Fabricius, Unternehmensrechtsreform und Mitbestimmung in einer sozialen Marktwirtschaft, 1982, S. 44.

56 Siehe $\S \S 14,31$ f. des Gesetzes über den Wertpapierhandel (WpHG) vom 26. Juli 1994; vgl. hierzu Hopt, ZHR 159 (1995), 135 ff.

57 Vgl. dazu R. Zeppernick, Transfer-Einkommen und Einkommensverteilung, 1986.

58 Fikentscher, Wirtschaftsrecht, Bd. II, 1983, S. 63; Stern a. a. O. (FN6), S. 899.

59 Böhm, WuW 1956, 173 ff., 186 f.; Nipperdey, WuW 1954, 211 ff., 223 f.

60 Zutreffend Rittner, Wirtschaftsrecht, 2. Aufl. 1987, S. 50; ebenso Schwark, FS Fabricius, Bochumer Beiträge zum Berg- und Energierecht, Bd. 7, 1989, S. 203, 204.

61 Wettbewerb als Aufgabe, 2. Aufl. 1947.

62 So E. R. Huber, Wirtschaftsverwaltungsrecht, Bd. 2, 2. Aufl. 1954, S. 203 f.; ebenso wieder Zacher a. a. O. (FN 10), S. 1081.

63 FIW-Schriftenreihe, Heft 120, S. $107 \mathrm{ff}$.

64 Dazu vgl. vor allem die Veröffentlichungen von v. Nell-Breuning, u. a. in HDSW 10, Stuttgart 1959 usw, S. 6 ff.; Fabricius a. a. O. (FN 55), S. $93 \mathrm{ff}$.

65 Vgl. Schlecht, Grundlagen und Perspektiven der sozialen Marktwirtschaft, 1990, S. 36 ff., 42 ff., 195 ff. u. passim. 
66 Zutreffend Müller-Volbehr, JZ 1982, 132, 138.

67 Ebenso Fikentscher a. a. O. (FN 58), S. 63.

68 Zur Wirkungsanalyse von Subventionen vgl. nur Möschel, Den Staat an die Kette legen - Gegen die Aushöhlung des Wettbewerbs durch den Staat, 1995, S. 34 ff.; kritisch Schwintowski, RabelsZ 1994, 232, 253 ff., $275 \mathrm{ff}$.

69 Stober, Handbuch des Wirtschaftsverwaltungs- und Umweltrechts, 1989, § 110 (S. 1219 f.).

70 Zur wenig effizienten Kontrolle durch Konkurrenten (Konkurrentenklage) Rinck/Schwark, 6. Aufl. Rdnr. 908 f.

71 Möschel a. a. O. (FN 68), S. 37.

72 Adam Smith, Der Wohlstand der Nationen, herausgegeben von Recktenwald, München 1974, 4. Buch, Zweites Kap., S. 371.

73 Dazu Ryball/Hofmann, NVwZ 1995, 230 ff.

74 Insbesondere von Arndt, JuS 1993, 360, 361 ff. und Hohmann, DÖV 1991, 191, 194; vgl. auch Selmer, VVDStR1 52, 10, 25 f. m. w. N.

75 A. a. O. (FN 74), S. 361; ebenso Selmer a. a. O. (FN 74), S. 26.

76 VVDStRl 52 (1993), S. 20 f. (Selmer); S. 166 (Schachtschneider).

77 So bereits von Münch, GG-Kommentar 1978, Bd. 3, Art. 72, Rdnr. 24; Gruson, Die Bedürfniskompetenz, Inhalt und Justitiabilität des Art. 72 Abs. 2 des GG, 1967, S. 48; Wengler, JZ 1965, 136; kritisch Degenhart, Staatsrecht I, 8. Aufl. 1992, Rdnr. 107.

78 VVDStR1 52, 71, 83; vgl. auch Selmer ebdt. S. 10, 19 f.

79 So auch Arndt, JuS 1993, 360, 362; kritisch Maunz in: Maunz/Dürig/Herzog, GG, Art. 72, Rdnr. 23; Bothe in: AK z. GG, Art. 72, Rdnr. 14; Stern, Handbuch des Staatsrechts, Bd. II, 1980, S. 596 f.

80 Aufschlußreiche Zahlen nennt Bayer, VVDStRl 52, S. 142.

81 Hinzuweisen ist auf die Normenkontrollanträge der Länder Bremen, Hamburg, Saarland und Schleswig-Holstein, über die BVerfG JZ 1992, 962 entschieden hat.

82 Dazu Arndt a. a. O. (FN 74), S. 360, 364.

83 Vgl. dazu das Gesetz über die Errichtung des Fonds ,Deutsche Einheit" vom 25.6.1990 (BGB1 II, S. 518, 533). Der Fonds wurde aufgrund des Staatsvertrages mit der DDR vom 18.5.1990 (BGB1 II, 537) errichtet.

84 Zum weiten Beihilfebegriff des EGV vgl. M. Schröder, ZHR 152 (1988), 391, 400 ff.; Müller-Graff, ZHR 152 (1988) S. 404, 411 ff.; Matthies, ebdt., S. 442 f.

85 Zum Schutz des unantastbaren Bestandes des deutschen GG (Art. 79 Abs. 3 GG), der auch das Sozialstaatsprinzip umfaßt, und zwar auch gegenüber EG-Recht, vgl. zuletzt das Maastricht-Urteil des BVerfG, 
BVerfGE 89, 155, 172, 182 (betreffend Demokratiegebot), 177 f. (betreffend Grundrechtsschutz).

86 Ebenso Wenig in: Groeben-Thiesing-Ehlermann, EWGV, 4. Aufl., Art. 92, Rdnr. 37.

87 Thiesing in: Groeben-Boeck-Thiesing-Ehlermann, EWGV, 3. Aufl., vor Art. 92, Rdnr. 6 - 9; Rengeling, JZ 1984, 795, 797.

88 Wenig a. a. O. (FN 86), Art. 92., Rdnr. 37.

89 Zu diesem Leitbegriff vor allem Forsthoff, Verwaltungsrecht, 10. Aufl. 1973, S. 370 m. w. N.; ders., Die Verwaltung als Leistungsträger, 1938.

90 Stern a. a. O. (FN 6), S. 897 f.

91 BVerwGE 39, $329 \mathrm{ff}$.

92 Schwark, FS Fabricius, a. a. O. (FN 60), S. 203, 215 f.

93 Stober, a. a. O. (FN 69), § 47 I, II (S. 597 f.).

94 Schwark, Auf einem dritten Weg (FS Ridder), 1989, 136, 138 f.

95 Ebenso Möschel a. a. O. (FN 68) unter Hinweis auf die regelmäßig unbegründeten Fiskusabwehrklagen vor den Verwaltungsgerichten.

96 Rinck/Schwark, Wirtschaftsrecht, 6. Aufl., Rdnr.254 ff.

97 BGHZ 82, 375, 395 ff. (zu Brillenselbstabgabestellen der Ortskrankenkassen), dazu Rinck/Schwark a. a. O. (FN 96), Rdnr. 670 ff.

98 Vgl. EuGH Rs. 41/83, Slg. 1985, 873 - British Telecom. Die Entscheidung wendet die Wettbewerbsregeln sogar auf legislative Akte an.

99 Zur darüber hinaus bestehenden Relevanz der effet-utile Rspr. des EuGH vgl. Möschel, Weiterentwicklung der Europäischen Gemeinschaften und der Marktwirtschaft, FIW-Schriftenreihe Heft 148, 1992, S. 89 ff. m. w. N.

100 So EuGH Rs. 41/90, Slg. 1991, 1979.

101 Zutreffend Pernice in: Grabitz/Hilf (Hrsg.), Kommentar zur EU, Bd. I, Art. 90, Rdnr. 51.

102 Richtlinie der Kommission über den Wettbewerb auf dem Gebiet der Telekommunikationsendgeräte, AB1. 1988 Nr. L 131, S. 73.

103 Hochbaum in: Groeben-Thiesing-Ehlermann, EWGV, 4. Aufl., Art. 84, Rdnr. 84.

104 Hochbaum a. a. O. (FN 103), Art. 90, Rdnr. 76.

105 Hochbaum a. a. O. (FN 103), Art. 90, Rdnr. 78.

106 Vgl. Pernice a. a. O. (FN 101), Rdnr. 37 mit Nachweisen für und wider.

107 Grünbuch über die Erreichung des gemeinsamen Marktes für den Rundfunk, insbesondere über Satellit und Kabel, KOM (84), 300 endg.

$108 \mathrm{Zu}$ den zaghaften Bemühungen, eine Sozialunion (ohne Großbritannien) zu schaffen, siehe nur Steindorff, Einl. Europarecht, 13. Aufl. 1995, S. XXIII $f$.

109 BVerfGE 21, 245, 251. 
110 Vgl. Scholz in: Maunz-Dürig, GG, Art. 12, Rdnr. 45: Essentielles Ordnungsanliegen der Sozialstaatlichkeit.

111 Scholz a. a. O. (FN 110), Art. 12, Rdnr. 46; Maunz ebdt. Art. 109, Rdnr. 29.

112 Vgl. auch Art. 2 EGV, der ein hohes Beschäftigungsniveau zu den Aufgaben der Gemeinschaft zählt.

113 Vgl. etwa Ballerstedt a. a. O. (FN 9), S. 84 ff.; Scholz in: Böckenförde/Jekewitz/Ramm, Soziale Grundrechte, 1981, S. 81; Stern a. a. O. (FN 5), S. 904; Zacher a. a. O. (FN 10), S. 1088.

114 Scholz, RdA 1993, 249, 251; Nebendahl, ZRP 1991, 257, 259; Gode, DVB1 1990, 1207, 1210.

115 A. a. O. (FN 17), Art. 20, IV, Rdnr. 71.

116 Zutreffend Scholz in: Maunz-Dürig, GG, Art. 12, Rdnr. 46.

117 Vgl. Teil 2, Art. 1 der europäischen Sozialcharta vom 18.10.1961; Teil 3 , Art. 6 der Konvention über wirtschaftliche, soziale und kulturelle Rechte vom 19.12.1966; Art. 1, Abs. 1 Übereinkommen Nr. 126 der ILO vom 9.6.1964; dazu Gode, DVB1 1990, 1207, 1211.

118 Vgl. Art. 23, Abs. 1 Allgemeine Erklärung der Menschenrechte vom 10.12.1948; dazu Gode a. a. O. (FN 116).

119 Vgl. BVerfGE 5, 85, 206; 7, 198, 205; 8, 115, 167; 21, 362, 372; 22, 180, 204; 35, 348, 355 f.; 40, 121, 183; 45, 376, 387; a. A. aber Kittner in: Böckenförde/Jekewitz/Ramm, Soziale Grundrechte, 1981, S. $92 \mathrm{f}$.

120 BAGE 48, 122, 138; 38, 69, 80; 2, 221, 224 f.; Wipfelder, VB1 BW 1990, 367, 369 m. w. N.; vgl. auch BVerfGE 50, 290, 362 und die päpstlichen Sozialenzykliken, dazu Fabricius a. a. O. (FN 55), S. 87 ff.

121 Scholz in: Böckenförde/Jekewitz/Ramm, Soziale Grundrechte, 1981, S. 75, 82; Kittner a. a. O. (FN 17), Art. 20, IV, Rdnr. 66.

122 Übersicht bei Scholz, RdA 1993, 249, 252 ff.; Wipfelder, VB1 BW 1990, $367 \mathrm{ff}$.

123 Scholz, RdA 1993, 249, 253, 257; Scholz-Anlehner, Arch. PT 1993, 103, 124 ff.; unzutreffend H.-P. Schneider, DÖV 1987, 749.

124 Kritisch Kittner a. a. O. (FN 17), Art. 20, IV, Rdnr. 72.

$125 \mathrm{Zu}$ den Vorzügen ,ökonomischer Intervention“ Zacher a. a. O. (FN 10), S. 1086.

126 Biedenkopf, Die neue Sicht der Dinge, 2. Aufl. 1985, S. 272 ff.; Frankfurter Institut für Wirtschaftspolitische Forschung: Mehr Markt im Arbeitsrecht, 1986, S. $16 \mathrm{ff}$.

127 Sog. neoklassische Sicht, die vor allem von Unternehmensseite vertreten wird.

128 Keynes, z. B. in The General Theory of Employment in: Collected Writings of J. M. Keynes, XIV, London, Basingstoke, 1973, S. 109 ff.; 
vgl. auch Gabler, Wirtschaftslexikon, 13. Aufl., Stichwort: Keynessche Lehre. Neben diesen Grundpositionen gibt es zahlreiche weitere Lehren.

129 Auskunft Frau Lücke, Bundesministerium für Arbeit und Sozialordnung, Bonn.

1301986 knapp 25\%, s. Frankfurter Institut a. a. O. (FN 126), S. 20.

131 BAG AP 11 zu $§ 111$ BetrVerfG 1972=ZIP 1983, 210 (Verlagerung vom Heidelberger Platz zur Otto-Suhr-Allee in Berlin).

132 FAZ-Magazin vom 19.1.1996, S. 42 f.

133 BMWi, Wirtschaft in Zahlen '95, S. 50.

134 So Koenigs, DB 1995, 1710; Gerken/Löwisch/Rieble, BB 1995, 2370, 2372 ff; a.A. Hanau, NJW 1969, 1369, 1371 ff.

$135 \S 42$ Abs. 4 S. 1 Nr. 2 BBG; vgl. Wolff/Bachof/Stober, Verwaltungsrecht II, 5. Aufl. § 113 Rdnr. 7.

136 Biedenkopf a. a. O. (FN 126), S. $331 \mathrm{f}$.

137 Hankel, Die sieben Todsünden der Vereinigung, 1993, S. 76.

138 Kittner, in: Böckenförde/Jekewitz/Ramm, Die sozialen Grundrechte, 1981, S. 92.

139 Reuter, RdA 1978, 344, 346.

140 Die protestantische Ethik und der Geist des Kapitalismus, Archiv für Sozialwissenschaft und Sozialpolitik 20 (1904), 1 ff. und 21 (1905), 1 ff., Neudruck 1993.

141 Ritter (Hrsg.), Historisches Wörterbuch der Philosophie, Bd. 1, 1971, Stichwort: Arbeit, Sp. 483; dort auch zu Thomas von Aquin, der die Arbeit nur naturali ratione notwendig zur Erhaltung des Lebens ansah und der ganz anderen Lehre des Aristoteles, wonach Arbeit den Menschen an der Muße der Kontemplation, dem eigentlichen Tun des Weisen, hindere. Arbeit und Tugend schlössen sich gegenseitig aus.

142 Das Kapital, Kritik der politischen Ökonomie, MEW 23 (1982), S. 49 ff., 57.

143 Die Aussagen von Marx gelten nämlich für jede Art von Arbeit, also auch die unbezahlte.

144 S. o. FN 141.

145 Mestmäcker, Über die normative Kraft privatrechtlicher Verträge, Münsteraner Antrittsvorlesung vom 13.11.1963, abgedr. in ders., Recht und ökonomisches Gesetz, 2. Aufl. 1984, S. 397, 398.

146 A. a. O. (FN 9), S. 56; vgl. auch Dürig, FS Nawiasky, S. 157, 168 ff.; unzutreffend BAG AP Nr. 2 zu $§ 13 \mathrm{KSchG}$.

147 A. a. O. S. 106; Fried legt einen Vertrag zugrunde, in dem ein Kleinunternehmer einen Arbeitnehmer zu einer gefährlichen Arbeit gegen geringe Bezahlung verpflichtet und darauf hinweist, die Sicherheits- 
vorkehrungen seien begrenzt. Der Arbeitnehmer fällt von einer wackeligen Leiter und verletzt sich schwer. Das Sozialversicherungsrecht der USA wird ausgeblendet.

148 So Zöllner, AcP 176 (1976), 221, 240 für den Arbeitsvertrag; SchmidtRimpler (AcP 147 (1941), 130 ff.) hat deshalb die Richtigkeitsgewähr zu Recht in der notwendigen Zustimmung des von einer unrichtigen (auch unsozialen) Willenserklärung Betroffenen gesehen.

149 Ballerstedt a. a. O. (FN 9); S. 59.

150 Gesetz-Sammlung für die Königlichen Preußischen Staaten, 1839, S. $156-158$

151 Überblick bei Larenz, Allgemeiner Teil des deutschen Bürgerlichen Rechts, 7. Aufl. 1989, § 3 II (S. 56 ff.); Jendrek in: Erman, BGB-Handkommentar, 9. Aufl. 1993, vor § $535 \mathrm{Rdnr} .70 \mathrm{ff}$.

152 Vgl. hierzu Krämer, Verbraucherschutz, in: Dauses, Handbuch des EG-Wirtschaftsrechts, H. V; Borchert, Verbraucherschutzrecht, 1994, S. 4.

153 Palandt-Heinrichs, BGB, 54. Aufl., § 134 Anm. 5b m. w. N.

154 Zutreffend Ballerstedt a. a. O. (FN 9), S. 57.

155 Aus der neueren Literatur vgl. vor allem Hönn, Kompensation gestörter Vertragsparität, 1982; Fastrich, Richterliche Inhaltskontrolle im Privatrecht, 1992; Limbach, JuS 1985, 10 ff.

156 BVerfGE 89, 214, $232 \mathrm{f}$.

157 Vgl. die Urteile des IX. Senats in BGHZ 106, 269; 107, 92; NJW 1991, 2015 ff.; a. A. der XI. Senat, NJW 1991, 923 und ZIP 1993, 26.

158 BVerfGE 5, 85, 206; 8, 274, 329; vgl. ferner BVerfGE 81, 242, 255.

159 Signifikant Anfang der 90-er Jahre, vgl. BMWi, Wirtschaft in Zahlen '95, S. $106-108$.

160 LG Göttingen, Urteil vom 3.2.1995, abgedr. in: Direkter Anlegerschutz, 8-9/1995, $253 \mathrm{ff}$.

161 BGH WM 1982, 862 f.=NJW 1982, 2823, 2824.

162 Die soziale Aufgabe des Privatrechts, Berlin 1889 (J. Springer). 


\section{Eberhard Schwark}

1939 in Hagen (Westf.) geboren.

1958 - 1962 Studium der Rechtswissenschaft an den Universitäten Freiburg, FU Berlin und Bonn.

1968 Zweite juristische Staatsprüfung.

1969 Promotion an der Juristischen Fakultät der Universität Bonn.

1969 - 1980 Referent im Bundesministerium für Wirtschaft, beim Internationalen Währungsfonds, Washington D. C. und im Bundesministerium der Justiz.

1978 Habilitation an der Universität Bonn.

1980 - 1981 Professor an der Universität Heidelberg.

1981 - 1993 Professor an der Ruhr-Universität Bochum.

Seit Herbst 1993 Professor für Bürgerliches Recht, Deutsches und Europäisches Handels- und Wirtschaftsrecht an der HumboldtUniversität zu Berlin.

Seit 1994 Geschäftsführender Direktor des Instituts für Bank- und Kapitalmarktrecht.

Ausgewählte Veröffentlichungen

Der Begriff der Allgemeinen Gesetze in Art. 5 Abs. 2 des Grundgesetzes. Berlin 1970.

Anlegerschutz durch Wirtschaftsrecht. München 1976.

Wirtschaftsrecht. 6. Aufl. Köln 1986.

Rechtsfragen des Konsumentenkredits. Frankfurt 1986.

Börsengesetz. Kommentar. 2. Aufl. München 1994.

Prospekthaftung und Kapitalerhaltung in der AG. In: Schmidt/ Schwark (Hrsg.). Festschrift Raisch. Köln 1995. 
In der Reihe Öffentliche Vorlesungen sind erschienen:

1 Volker Gerhardt: Zur philosophischen Tradition der Humboldt-Universität

2 Hasso Hofmann: Die versprochene Menschenwürde

3 Heinrich August Winkler: Von Weimar zu Hitler Die Arbeiterbewegung und das Scheitern der ersten deutschen Demokratie

4 Michael Borgolte: ,Totale Geschichte” des Mittelalters? Das Beispiel der Stiftungen

5 Wilfried Nippel: Max Weber und die Althistorie seiner Zeit

6 Heinz Schilling: Am Anfang waren Luther, Loyola und Calvin ein religionssoziologisch-entwicklungsgeschichtlicher Vergleich

7 Hartmut Harnisch: Adel und Großgrundbesitz im ostelbischen Preußen 1800 - 1914

8 Fritz Jost: Selbststeuerung des Justizsystems durch richterliche Ordnungen

9 Erwin J. Haeberle: Historische Entwicklung und aktueller internationaler Stand der Sexualwissenschaft

10 Herbert Schnädelbach: Hegels Lehre von der Wahrheit

11 Felix Herzog: Über die Grenzen der Wirksamkeit des Strafrechts

12 Hans-Peter Müller: Soziale Differenzierung und Individualität Georg Simmels Gesellschafts- und Zeitdiagnose

13 Thomas Raiser: Aufgaben der Rechtssoziologie als Zweig der Rechtswissenschaft

14 Ludolf Herbst: Der Marshallplan als Herrschaftsinstrument? Überlegungen zur Struktur amerikanischer Nachkriegspolitik

15 Gert-Joachim Glaeßner: Demokratie nach dem Ende des Kommunismus

16 Arndt Sorge: Arbeit, Organisation und Arbeitsbeziehungen in Ostdeutschland 
17 Achim Leube: Semnonen, Burgunden, Alamannen

Archäologische Beiträge zur germanischen Frühgeschichte

18 Klaus-Peter Johne: Von der Kolonenwirtschaft zum Kolonat

Ein römisches Abhängigkeitsverhältnis im Spiegel der Forschung

19 Volker Gerhardt: Die Politik und das Leben

20 Clemens Wurm: Großbritannien, Frankreich und die westeuropäische Integration

21 Jürgen Kunze: Verbfeldstrukturen

22 Winfried Schich: Die Havel als Wasserstraße im Mittelalter: Brücken, Dämme, Mühlen, Flutrinnen

23 Herfried Münkler: Zivilgesellschaft und Bürgertugend Bedürfen demokratisch verfaßte Gemeinwesen einer sozio-moralischen Fundierung?

24 Hildegard Maria Nickel: Geschlechterverhältnis in der Wende Individualisierung versus Solidarisierung?

25 Christine Windbichler: Arbeitsrechtler und andere Laien in der Baugrube des Gesellschaftsrechts Rechtsanwendung und Rechtsfortbildung

26 Ludmila Thomas: Rußland im Jahre 1900 Die Gesellschaft vor der Revolution

27 Wolfgang Reisig: Verteiltes Rechnen: Im wesentlichen das Herkömmliche oder etwa grundlegend Neues?

28 Ernst Osterkamp: Die Seele des historischen Subjekts Historische Portraitkunst in Friedrich Schillers „Geschichte des Abfalls der vereinigten Niederlande von der Spanischen Regierung"

29 Rüdiger Steinlein: Märchen als poetische Erziehungsform Zum kinderliterarischen Status der Grimmschen „Kinder-und Hausmärchen”

30 Hartmut Boockmann: Bürgerkirchen im späteren Mittelalter

31 Michael Kloepfer: Verfassungsgebung als Zukunftsbewältigung aus Vergangenheitserfahrung

Zur Verfassungsgebung im vereinten Deutschland

32 Dietrich Benner: Über die Aufgaben der Pädagogik nach dem Ende der DDR 
33 Heinz-Elmar Tenorth: „Reformpädagogik”

Erneuter Versuch, ein erstaunliches Phänomen zu verstehen

34 Jürgen K. Schriewer: Welt-System und Interrelations-Gefüge Die Internationalisierung der Pädagogik als Problem Vergleichender Erziehungswissenschaft

35 Friedrich Maier: „Das Staatsschiff” auf der Fahrt von Griechenland über Rom nach Europa

$\mathrm{Zu}$ einer Metapher als Bildungsgegenstand in Text und Bild

36 Michael Daxner: Alma Mater Restituta oder Eine Universität für die Hauptstadt

37 Konrad H. Jarausch: Die Vertreibung der jüdischen Studenten und Professoren von der Berliner Universität unter dem NS-Regime

38 Detlef Krauß: Schuld im Strafrecht

Zurechnung der Tat oder Abrechnung mit dem Täter?

39 Herbert Kitschelt: Rationale Verfassungswahl?

Zum Design von Regierungssystemen in neuen Konkurrenzdemokratien

40 Werner Röcke: Liebe und Melancholie

Formen sozialer Kommunikation in der 'Historie von Florio und Blanscheflur'

41 Hubert Markl: Wohin geht die Biologie?

42 Hans Bertram: Die Stadt, das Individuum und das Verschwinden der Familie

43 Dieter Segert: Diktatur und Demokratie in Osteuropa im 20. Jahrhundert

44 Klaus R. Scherpe: Beschreiben, nicht Erzählen! Beispiele zu einer ästhetischen Opposition: Von Döblin und Musil bis zu Darstellungen des Holocaust

45 Bernd Wegener: Soziale Gerechtigkeitsforschung: Normativ oder deskriptiv?

46 Horst Wenzel: Hören und Sehen - Schrift und Bild Zur mittelalterlichen Vorgeschichte audiovisueller Medien

47 Hans-Peter Schwintowski: Verteilungsdefizite durch Recht auf globalisierten Märkten

Grundstrukturen einer Nutzentheorie des Rechts 
48 Helmut Wiesenthal: Die Krise holistischer Politikansätze und das Projekt der gesteuerten Systemtransformation

49 Rainer Dietrich: Wahrscheinlich regelhaft. Gedanken zur Natur der inneren Sprachverarbeitung

50 Bernd Henningsen: Der Norden: Eine Erfindung Das europäische Projekt einer regionalen Identität

51 Michael C. Burda: Ist das Maß halb leer, halb voll oder einfach voll? Die volkswirtschaftlichen Perspektiven der neuen Bundesländer

52 Volker Neumann: Menschenwürde und Existenzminimum

53 Wolfgang Iser: Das Großbritannien-Zentrum in kulturwissenschaftlicher Sicht

Vortrag anläßlich der Eröffnung des Großbritannien-Zentrums an der Humboldt-Universität zu Berlin

54 Ulrich Battis: Demokratie als Bauherrin

55 Johannes Hager: Grundrechte im Privatrecht

56 Johannes Christes: Cicero und der römische Humanismus

57 Wolfgang Hardtwig: Vom Elitebewußtsein zur Massenbewegung Frühform des Nationalismus in Deutschland 1500 - 1840

58 Elard Klewitz: Sachunterricht zwischen Wissenschaftsorientierung und Kindbezug

59 Renate Valtin: Die Welt mit den Augen der Kinder betrachten Der Beitrag der Entwicklungstheorie Piagets zur Grundschulpädagogik

60 Gerhard Werle: Ohne Wahrheit keine Versöhnung! Der südafrikanische Rechtsstaat und die Apartheid-Vergangenheit

61 Bernhard Schlink: Rechtsstaat und revolutionäre Gerechtigkeit. Vergangenheit als Zumutung? (Zwei Vorlesungen)

62 Wiltrud Gieseke: Erfahrungen als behindernde und fördernde Momente im Lernprozeß Erwachsener

63 Alexander Demandt: Ranke unter den Weltweisen; Wolfgang Hardtwig: Die Geschichtserfahrung der Moderne und die Ästhetisierung der Geschichtsschreibung: Leopold von Ranke (Zwei Vorträge anläßlich der 200. Wiederkehr des Geburtstages Leopold von Rankes ) 
64 Axel Flessner: Deutsche Juristenausbildung

Die kleine Reform und die europäische Perspektive

65 Peter Brockmeier: Seul dans mon lit glacé - Samuel Becketts Erzählungen vom Unbehagen in der Kultur

66 Hartmut Böhme: Das Licht als Medium der Kunst. Über Erfahrungsarmut und ästhetisches Gegenlicht in der technischen Zivilisation

67 Sieglind Ellger-Rüttgardt: Berliner Rehabilitationspädagogik: Eine pädagogische Disziplin auf der Suche nach neuer Identität

68 Christoph G. Paulus: Rechtsgeschichtliche und rechtsvergleichende Betrachtungen im Zusammenhang mit der Beweisvereitelung 